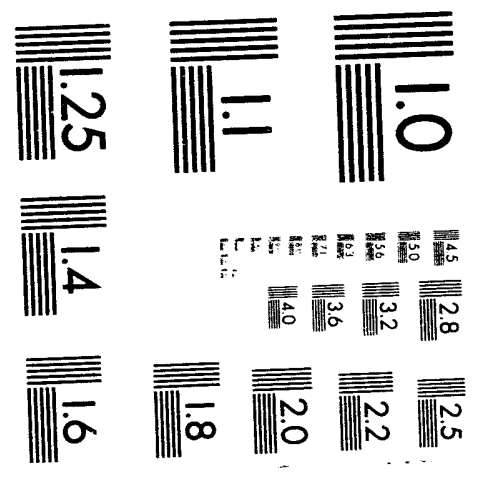



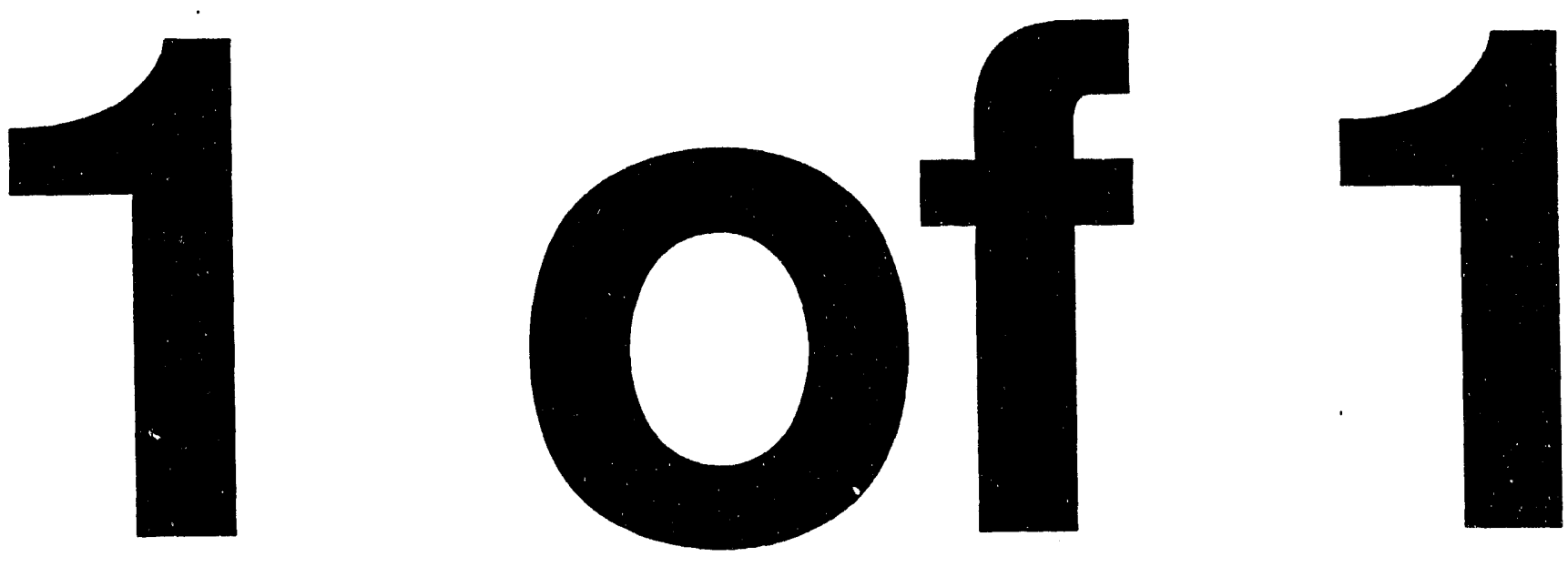


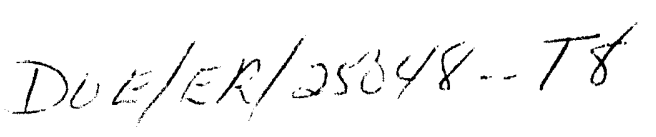

\title{
PARALLEL SUPERCOMPUTING: ADVANCED METHODS, ALGORITHMS, AND SOFTWARE FOR LARGE-SCALE LINEAR AND NONLINEAR PROBLEMS
}

\author{
G.F. Carey, Director \\ Computational Fluid Dynamics Laboratory
}

D.M. Young, Director

Center for Numerical Analysis

\section{DISCLAIMER}

This report was prepared as an account of work sponsored by an agency of the United States Government. Neither the United States Government nor any agency thereof, nor any of their employees, makes any warranty, express or implied, or assumes any legal liability or responsibility for the accuracy, completeness, or usefulness of any information, apparatus, product, or process disclosed, or represents that its use would not infringe privately owned rights. Reference herein to any specific commercial product, process, or service by trade name, trademark, manufacturer, or otherwise does not necessarily constitute or imply its endorsement, recommendation, or favoring by the United States Government or any agency thereof. The views and opinions of authors expressed herein do not necessarily state or reflect those of the United States Government or any agency thereof. 


\section{TABLE OF CONTENTS}

Page No.

1. Summary 3

2. Overview of Research 3

2.1 Background and Motivation 3

2.2 Research Objectives 4

2.3 Personnel and Personnel Organization 5

2.4 Interaction and Dissemination 5

3. Description of the Research 5

3.1 Domain Decomposition Techniques 6

3.2 Spectral and Hierarchic p Schemes 6

3.3 Element-by-Element Parallel Strategies 7

3.4 Multilevel/Multigrid Decomposition 8

3.5 Iterative Methods for the Solution of Large Sparse Systems of Linear 9 Algebraic Equations

3.6 Adaptive Parameter Determination and Error Purification 9

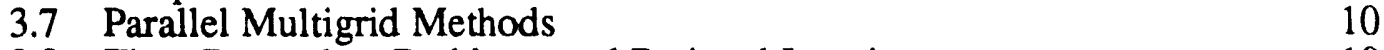

3.8 Time Dependent Problems and Rational Iteration 10

$\begin{array}{ll}3.9 \text { Nonsymmetric Systems } & 12\end{array}$

$\begin{array}{ll}3.10 \text { Software Research } & 13\end{array}$

$\begin{array}{ll}3.11 \text { Applications } & 15\end{array}$

$\begin{array}{ll}3.12 \text { References } & 18\end{array}$

4. Institutional Commitment and Other Support 24

5. Impact on Infrastructure of Science/Engineering 24

6. Budget 25

6.1 Budget Period Summary 25

6.2 Project Period Summary 26

$\begin{array}{ll}\text { 7. Budget Justification } & 28\end{array}$

8. Statement of Other Support 28

$\begin{array}{ll}\text { 9. Assurances } & 28\end{array}$

10. Statement of Unobligated Funds 28

11. Biographical Data 29

G. F. Carey

D. M. Young

D. R. Kincaid

K. Sepehrnoori

\section{APPENDIX}




\section{SUMMARY}

The program outlined here is directed to research on methods, algorithms, and software for distributed parallel supercomputers. Of particular interest are finite element methods and finite difference methods together with sparse iterative solution schemes for scientific and engineering computations of very large-scale systems. Both linear and nonlinear problems will be investigated. In the nonlinear case, applications with bifurcation to multiple solutions will be considered using continuation strategies. The parallelizable numerical methods of particular interest are a family of partitioning schemes embracing domain decomposition, element-byelement strategies, and multi-level techniques. The methods will be further developed incorporating prarallel iterative solution algorithms with associated preconditioners in parallel computer soffware. The schemes will be implemented on distributed memory parallel architectures such as the CRAY MPP, Intel Paragon, the NCUBE3, and the Connection Machine. We will also consider other new architectures such as the Kendall-Square (KSQ) and proposed machines such as the TERA. The applications will focus on large-scale three-dimensional nonlinear flow and reservoir problems with strong convective transport contributions. These are legitimate grand challenge class computational fluid dynamics (CFD) problems of significant practical interest to DOE. The methods developed and algorithms will, however, be of wider interest.

The research will be led by Dr. G.F. Carey (Director, Computational Fluid Dynamics Laboratory) and Dr. D.M. Young (Director, Center for Numerical Analysis). Dr. Carey and Dr. Young are internationally known for their work on finite elements and iterative methods, respectively, and these topics are central to the proposed research. Dr. D. Kincaid (Computer Sciences), Dr. K. Sepehnoori (Petroleum Engineering) and Dr. R. McLay (Computational Mechanics) will participate as co-investigators in the research program. This program will also support graduate students and post-doctoral training in this rapidly evolving area. The research builds on our current efforts in developing methods, algorithms and software for advanced architectures. Large scale applications include, in particular, three dimensional Navier-Stokes calculations and reservoir simulation. We have received awards for our work on high performance computing and large-scale applications related to the proposed research. A list of related reports and publications are given later.

The research will be facilitated by a recent (1992) grant of an Intel distributed processor to Dr. Carey and other researchers at UT. We also anticipate that the UT Center for High Performance Computing which now has a CRAY-YMP will also acquire a distributed memory supercomputer during the proposed period of funding. The proposed research also receives support in the form of supercomputer access at several national facilities. In particular, we have been working closely with the Parallel Research Groups at Sandia and Los Alamos and have ongoing studies using the NCUBE and CM Machines at these sites.

\section{OVERVIEW OF THE RESEARCH}

\subsection{Background and Motivation}

Conventional serial computers are limited by certain factors such as the need to dissipate heat and the difficulty of fabricating sub-micron devices at significantly smaller scales. Short of major breakthroughs using radical new technologies (e.g., superconductors or optics), the traditional approach of miniaturization is reaching a point of diminishing returns. This, together with the economics of chip production, has led to the evolution of several parallel and parallelvector architectures. These range from shared-memory supercomputers of coarse granularity (such as the CRAY Y-MP) to massively parallel distributed memory architectures (such as the Connection Machine, Intel Paragon, NCUBE and CRAY-MPP).

A major use of these advanced scientific computers is for the approximate solution of linear and nonlinear partial differential equations (PDE's) describing natural processes and 
characterizing engineering design systems. Typically, the discretization process leads to largescale computationally-intensive sparse linear and nonlinear algebraic systems. For realistic 3D applications of engineering and scientific interest, many of these problems are beyond the scope of conventional computer systems.

Hardware advances have attained the point where powerful parallel and vector-parallel computers are now emerging that can provide multi-Gigaflop speeds (billions of floating-point operations per second). However, the effective exploitation of these machines for science and engineering applications necessitates a radical change in the way we think about numerical methods and algorithms (e.g., see Young [1989]). So far, the rapid progress in hardware developments has understandably overshadowed research on parallel numerical methods, algorithms, and software. Promotion of basic research in these areas is essential; otherwise the realization of the full potential of these new hardware designs will be unnecessarily delayed. Moreover, scientific software for parallel architectures must be developed as soon as possible to accelerate transition of basic research discoveries to the user community. The present research is directed to these issues.

\subsection{Research Objectives}

The focus of the proposed research concerns parallel methods, algorithms, and software for complex applications such as those in coupled fluid flow and heat transfer. It is directed principally toward the solution of large-scale PDE problems using iterative solvers for finite differences and finite elements on advanced computer architectures. This work embraces parallel domain decomposition, element-by-element, spectral, and multilevel schemes with adaptive parameter determination, rational iteration and related issues. Details are given in Section 3.

In addition to the fundamental questions related to developing new methods and mapping these to parallel computers, there are important software issues. Our group has played a significant role in the development of software both for iterative solvers and also for finite element codes. In particular, the ITPACK Project (Drs. Young and Kincaid) led to the development of several packages of iterative schemes and accelerators that are widely used in the scientific community. Recent vectorized versions of ITPACK and a derivative for nonsymmetric systems, NSPCG, have been used successfully by industry for device modeling, reservoir simulation, computational fluid dynamics and many other applications. We are extending the algorithms and software to parallel systems using the concept of iterative "kernels." This algorithm/software development is to be incorporated with "intelligent" finite difference and finite element schemes with adaptive refinement capabilities that permit the grid and solution to evolve concurrently.

Our recent research in computational fluid dynamics (CFD) has led to sustained multiGigaflop performance rates for parallel-vector computations of realistic large scale applications (not computational kernels alone). The main application areas for these performance studies have been two-dimensional problems in CFD. In particular, we have focused on Navier-Stokes problems (Dr. Carey) and on chemical-recovery-reservoir simulation (Dr. Sepehrnoori). Threedimensional applications will be central to the new work. The chemical flood simulator is based on our software UTCHEM, which now' is being used extensively in the Petroleum industry and is being extended to a parallel distributed environment. In our prototype Navier-Siokes program NSFLO on the CRAY Y-MP, an artificial timestep scheme is developed for integration to a steady state using domain decomposition. This program is being extended to the C-90, KSQ and TERA architecture families. It will also be developed for our INTEL hypercube and similar configurations.

Part of the work will focus on large scale three-dimensional applications and on massively parallel systems. With current systems capable of multi-Gigaflop performance, massively parallel systems may potentially deliver an order of magnitude improvement soon, and we are witnessing progress towards a Teraflop (a trillion floating-point operations per second) which will permit direct 3D Navier Stokes simulation, complex 3D simulation of natural processes such 
as global warming, full integrated design of engineering systems (reactors, solar exchangers, etc.), and 3D multicomponent-multiphase reservoir and containment transport simulation.

\subsection{Personnel and Personnel Organization}

The research involves collaboration of principal investigators in Computer Sciences, Mathematics, and Computational Fluid Dynamics. The program will be coordinated by Dr. G.F. Carey, (Director, Computational Fluid Dynamics Laboratory), and Dr. D.M. Young, (Director, Center for Numerical Analysis), as principal investigators. Dr. K. Sepehrnoori (Petroleum Engineering) and Dr. D. Kincaid (Computer Sciences) will participate as co-investigators. Dr. Sepehrnoori is developing methods for 3-D reservoir simulation in enhanced oil recovery and Dr. Kincaid is developing iterative Basic Linear Algebra Subroutine (BLAS) kernels for transportable vector-parallel iterative software. Dr. R. McLay (TICOM) will be involved in 3D parallel finite element computations for viscous Iow and heat transfer. This work will be carried out jointly with Dr. Carey for applications to manufacturing processes. A postdoctoral research fellow, as well as graduate research assistants, will also be supported. This support will allow us to continue our activities both on the algorithms and the software at a critical time in the development of this research area, since new and expanded hardware capabilities are becoming more widely available.

\subsection{Interaction and Dissemination}

During the past three years, we have conducted a joint seminar on "finite elements, iterative methods, and advanced computers" associated with our research program. This, together with regular discussion meetings between the investigators and graduate students, permits close coordination of our joint research. The investigators also jointly serve on related $\mathrm{PhD}$ committees and work closely with the graduate students on this research. A workshop on supercomputing was sponsored by us and held in Austin in conjunction with a conference on Iterative Methods honoring Dr. Young. These efforts have led to archival volumes and special journal issues related to the research. Dr. Young and Dr. Carey organized a workshop on Iterative Methods for Nonsymmetric Systems (Jan. 1992) in conjunction with the conference in Austin on Approximation Theory. A special journal issue on Multigrid methods co-edited by Drs. Carey and Mandel recently appeared (Dec. 1992). There is a close interaction with the parallel applications groups at Sandia and Los Alamos. Research contact with industrial associates also aids in dissemination of new material. Part of the proposed research dealing with manufacturing applications will be coordinated with Dr. David Gartling at Sandia.

\section{DESCRIPTION OF THE RESEARCH}

The focal points of the proposed research during the next three-year period involve parallel gradient-based solution strategies, parallel domain decomposition, parallel multilevel schemes, parallel local timestepping and asynchronous parallel strategies. Supporting software will be developed for both structured grids with finite differencing and unstructured grids for finite elements with parallel and parallel-vector iterative solution methods. Related issues involve some aspects of element-by-element and matrix-free methods, transient techniques and rational iteration, additive correction and nonsymmetric systems. Linear and nonlinear problems will be investigated. The research will primarily deal with distributed parallel-vector systems such as the Intel Paragon, NCUBE3, Connection Machine and CRAY MPP. However, other new parallel supercomputers such as the Kendall-Square (KSQ) Supercomputer and machines with data that "streams through memory" such as the proposed TERA will also be considered. Of particular interest are iterative methods, artificial transient techniques, nonsymmetric systems, and high $p$-spectral elements for linear and nonlinear three-dimensional applications. Consequently, we will be developing and applying new methods to 3D engineering applications of significant practical interest. These include 3D Navier Stokes, 3D reservoir simulation, 3D 
contaminant transport for hazardous waste, and 3D flow and transport for industrial processes. Another component of the research is the development of parallel basic iterative "kernels" to facilitate a modular software design. Further details of the research program are given in the following subsections.

\subsection{Domain decomposition techniques}

Domain decomposition techniques are closely tied to the partitioning of the domain on which the PDE is defined. Subdomains may themselves consist of a large number of elements in the finite-element, finite volume, or finite-difference discretization. In a finite element analysis, the mathematical formulation is based on a weak integral statement of the PDE problem. If this is restructured over the subdomains, then "natural" interface jump conditions apply across the interior boundaries between subdomains. This restructuring of the mathematical problem supports several opportunities for developing accelerated parallel iterative algorithms over the subdomains. For example, large contiguous subdomains can be distributed to parallel processors in a "divide-and-conquer" strategy with block-iterative solution techniques introduced on the subdomains. The interface relation provides the connection between subdomain block iteration, block preconditioning strategies, and effective supercomputer techniques. Families of methods are being developed for both overlapping and non-overlapping parallel domain decomposition. In particular, some of our recent work on superconvergent flux post-processing (Carey et al 1992) is being reformulated to apply to the interfaces of the subdomain problems in the nonoverlapping algorithm. This promises to yield faster convergence for both the serial and parallel schemes. Hierarchic imbeddings of grids can be exploited for both the domain decomposition schemes and the parallel multigrid methods, as described later.

We are developing parallel grid generation and optimization schemes based on domain decomposition. Grid generation for complex 2- and 3-dimensional domains has become a major "bottleneck" in large scale computations. At a meeting on grid generation, Paul Kutler of NASA Ames observed that "preparation of a good grid for large-scale shuttle-aerodynamic calculations requires a man-month or more" and that grid optimization calculations can rival the actual flow solution of the associated PDE's. We have developed an approach for local grid generation and optimization in a parallel system. By block iteration, an "optimal" local grid can be generated over the distributed processors and, moreover, the flow solution can be constructed concurrently with the grid. There are several open questions related to subdomain partitioning algorithms and load balance.

The present research will explore this domain decomposition work for distributed systems. We have access to the Connection machine at the Schlumberger Computer Research Group in Austin and at Los Alamos. Our collaborative work with the parallel applications group at Sandia is being coordinated with Dr. D. Cline (Sandia) who has been working with Dr. Carey and a graduate student (Alan Stagg) on parallel parabolized Navier Stokes computations for grand challenge applications on the NCUBE using domain decomposition. We plan to expand this activity to the Intel Paragon next year.

\subsection{Spectral and hierarchic $p$ schemes}

Some of the proposed finite element research involves adaptive mesh and spectral elements so called " $h$ " and " $p$ " methods. The standard finite-element error-estimates for regular problems have the form (Oden and Carey, 1985)

$$
\|e\|_{m} \leq C h^{p}
$$

where $C=C(p)$ and the rate $p$ depends on the polynomial degree of the basis; e.g., $p=k-m$, where $k$ is the polynomial degree. Here $\|.\|_{m}$ denotes the $H^{m}$ norm and $e=u-u_{h}$ is the approximation error for a discretizar: on with characteristic mesh size $h$. The error can be reduced by decreasing 
$h$ or increasing $k$ (or both). Clearly, for regular problems, the error reduction is greater if the polynomial degree is increased $(C(p)$ also decreases as $\mathrm{p}$ increases). This idea is the basis for high-degree spectral elements. A second consequence is that the element matrices are larger and more dense. Hence fewer elements are needed per subdomain to achieve the same accuracy; e.g. in 3-D, a tensor-product basis of degree 7 generates $2^{9}$ nodes per element for a scalar problem so the dense element matrices are of size $2^{9} \times 2^{9}$ for the scalar field problem. For less regular problems, the power $p$ in (1) is limited by the solution smoothness and increasing $p$ will not improve the global asymptotic rate. Here both mesh grading and polynomial enrichment are used. This will complicate the data structure.

We can develop a hierarchic multilevel $p$ scheme by introducing a hierarchic basis. In an hierarchic family, all lower-degree bases are nested as $p$ increases. This implies that new element matrices can be constructed by simply adding a row and column border to the current $(p-1)$ matrix. There are some interesting opportunities for economizing the element calculations that we also plan to pursue here. Moreover, the structure of the bordered submatrix systems can be exploited in associated gradient iterative schemes. Also note that the projection from the high level operator to lower levels simply involves removing rows and columns from the element matrices. We discuss this further in the treatment of element-by-element parallel schemes below.

\subsection{Element-by-Element Parallel Strategies}

In a previous phase of our work on subdomain techniques, we made an important breakthrough in the area of abstract domain decomposition techniques and iterative methods. This is in the form of an element-by-element scheme together with conjugate-gradient-like iterative solution of the finite element system for PDE problems. Indeed, these ideas are now becoming widely adopted for both serial and parallel computation. The essential idea in the element-by-element approach is to utilize the independent element calculations in the finite element approximation to parallelize the method. Moreover, appropriate iterative methods such as block-preconditioned conjugate gradient schemes can be recast at the element level to define a powerful new method in which much of the solution step can be parallelized. This method relies on the observation that conjugate-gradient-iterative methods involve repeated matrix-vector products and inner products. Rather than assembling the element contributions in the traditional fashion and then solving the merged sparse system, we write

$$
\sum_{e=1}^{E} \hat{A}_{e}=\sum_{e=1}^{E} C_{e}^{T} A_{e} C_{e}
$$

where $C_{c}$ is the Boolean transformation defining a map between local element numbering and the global node system. Here $e$ is the element index, $A_{e}$ is the dense local element matrix and $\hat{A}_{e}$ is the element matrix expanded to global matrix system size. In the subsequent matrix-vector products of the gradient iterative method, we can write

$$
v=A w=\left(\sum_{e=1}^{E} \hat{A}_{e}\right) w=\left(\sum_{e=1}^{E}\left(C_{e}^{T} A_{e} C_{e} w\right)\right)=\sum_{e=1}^{E} C_{e}^{T} A_{e} w_{e}=\sum_{e=1}^{E} C_{e}^{T} \nu_{e}
$$

It follows that the global matrix product can be carried out at the element level as $A_{e} w_{e}=v_{e}$, thus circumventing the usual matrix assembly step. More importantly, since the element contributions can be evaluated independently and in parallel, this implies that parallel and parallel-vector schemes can be developed from this framework in a natural and elegant manner. Further details are given in Carey [1993]. There are some important issues that arise in 
connection with communication on distributed systems and memory conflicts on shared parallel systems that warrant further study. For example, the extraction operation $w_{e}=C_{e} w$ must distribute parts of the global vector to respective processors. Similarly, the residual accumulation of element vectors to a global vector requires special attention.

One of the key features of the element-by-element approach is the fact that it is posed on an element-based data structure rather than a node-based structure. This implies that irregular unstructured grids can be utilized that may employ elements of different types, e.g., singular elements can be used near a fracture tip, polynomial (spectral) elements in the main field and "infinite" elements in the far-field. The method, therefore, lends itself to general parallel utilization and can be applied in conjunction with adaptive refinement to highly unstructured grids. This is particularly important since the preceeding approach may be combined with adaptive grid refinement to provide more efficient solution schemes for large-scale 3D applications. In the adaptive refinement procedure, the solution is computed on a coarse grid, an a posteriori analysis permits construction of error indicators that locate regions where grid refinement is warranted, and then the grid cells in these regions are locally refined. The solutionrefinement process is repeated until a desired accuracy is met.

The key ingredient here is the data structure that supports the refinement process. We have developed a scheme whereby quadrilateral or triangular elements are subdivided to quartets of sub-elements to generate a quadtree (Carey et al, 1988). The procedure can be extended to 3-D in the form of an octree data structure that is more complex and will be investigated as part of the proposed research. Since we are able to parallelize over elements in a block iterative solver, this implies that the scheme should be applicable to unstructured adaptive grids with little degradation in parallel performance. We also propose to extend this approach to nested (hierarchic) bases in which the polynomial degree is increased automatically to meet a desired solution accuracy. One of the main difficulties associated with $p$ methods is the significant degradation in conditioning as the element degree is increased. This has led to renewed interest in the selection of basis functions and the development of element block preconditioners that utilize special bases. We are currently investigating basis transformation techniques as a mechanism for generating good preconditioners within the framework of the element-by-element spectral methods (Barragy and Carey, 1991).

\subsection{Multilevel/Multigrid Decomposition}

The need to treat 3D problems has also motivated some of our work on hierarchic elements and multilevel schemes. We propose to implement the multilevel strategy in conjunction with both the spectral element procedure (multi-p) and also develop a parallel hierarchic $h$ scheme. In the hierarchic method, a macro element is repeatedly refined to produce a nested hierarchy of local refinements but, as in the $p$ scheme, the $h$-basis is constructed in a hierarchy rather than in the usual Lagrange manner. This leads to a nesting of matrix families in which rows and columns are simply added as the mesh is refined or "turned off" as the mesh is coarsened. The hierarchic $h$-method can be utilized in a multigrid-multilevel algorithm and the effect of the multigrid projections is to achieve a preconditioning so that the number of iterations of the parallel solver is independent of the mesh size $h$. (Of course, the work per solve still scales with the matrix size so scalability is still an issue.) Most of our work to date has involved 2D model problems. In the proposed work, the emphasis will be on the 3D case. The extension from 2D to 3D is far from routine; e.g., in 2D a quadrilateral element has four edge neighbors and four vertex neighbors whereas a "quadrilateral brick" in 3D has 6 face neighbor elements, 12 edge neighbors and 8 vertex neighbors. The situation for unstructured $3 \mathrm{D}$ tetrahedral grids is more complex. This implies parallel communication will be a more significant issue in $3 \mathrm{D}$.

Another difficulty with the 3D problem is that, even for single element subdomains, the memory requirements may be too demanding for some distributed systems. To circumvent this, we propose to develop and study element matrix-free schemes. That is, by using the tensor product structure of the finite element basis and the associated Gaussian quadrature, we are able to restructure the element calculations and the steps in the gradient iterative scheme to be less 
demanding with respect to local memory requirements for the distributed system. These ideas can also be applied in the context of time-dependent techniques.

Parallel preconditioning accelerators will be developed and tested as part of the study. In the proposed research, we plan to continue this work in the context of spectral finite-elements and hierarchic $h$ schemes with element-by-element domain decomposition as indicated previously. Additional work on transient methods has been initiated and is directed to both stable timeaccurate methods and also stable steady solution (not time accurate). More details are given later.

\subsection{Iterative Methods for Large Sparse Systems of Linear Algebraic Equations}

At the core of many problems involving the solution of partial differential equations is the problem of solving multiple systems of linear algebraic equations. Typically, the coefficient matrices for such systems are very large and very sparse. Our research is concerned with the development of rapidly converging iterative methods which make maximum use of highly parallel computer architectures such as the Connection Machine, the Intel Paragon and the Cray MPP.

There are two levels of parallelism to be considered in designing algorithms and programs for highly parallel computer systems. At one level we have "low level" or fine grain parallelism which is needed to ensure that each individual iteration is carried out efficiently. To do this one must consider questions of sparse data structures and the efficient manipulation of vectors and sparse matrices. In addition to this there is a need to consider "high level" parallelism which involves designing the overall iterative algorithm to obtain rapid convergence and, at the same time, retaining the fine grain parallelism.

Many of the standard iterative methods parallelize well but their convergence rates of ten leave something to be desired. For example, the use of a basic iterative method, such as the Jacobi method, combined with Chebyshev acceleration or conjugate gradient acceleration typically requires on the order of $K(A)^{1 / 2}$ iterations, where $K(A)$ is the condition of the matrix of the linear system. (For a typical elliptic equation defined over a grid of size $h,(K(A))^{1 / 2}$ is in the order of $h^{-1}$.) On the other hand, the accelerated Jacobi method is highly parallelizable. With some basic iterative methods, such as the accelerated SSOR method, it is sometimes possible to get $\mathrm{O}\left(h^{-1 / 2}\right)$, convergence if one uses the "natural" ordering of the grid points. However, the use of that ordering degrades parallelism. If one uses the "red-black" ordering of the grid points instead of the natural ordering, the accelerated SSOR method is highly parallel but the number of iterations increases from $\mathrm{O}\left(h^{-1 / 2}\right)$ to $\mathrm{O}\left(h^{-1}\right)$.

For certain types of problems, including "separable" elliptic problems in two- and threedimensions, one can often achieve convergence in $\mathrm{O}(\log K(A))$, or $\mathrm{O}\left(\log h^{-1}\right)$ iterations by the use of alternating direction implicit methods (ADI methods). While the commutativity of certain matrices is required to rigorously guarantee the rapid convergence, the method actually works well in many cases where the commutativity does not hold. To carry out the method requires the solution of a large number of tridiagonal linear systems. However, the solution of these systems can be carried out in parallel. Unfortunately, the ADI method is less robust than many other methods and often does not work well for problems involving coefficients which are discontinuous or highly variable.

\subsection{Adaptive Parameter Determination and Error Purification}

Conjugate gradient acceleration leads to more rapid convergence than Chebyshev acceleration. However, with conjugate gradient acceleration several inner products must be evaluated for each iteration. This is a disadvantage with distributed memory computers since inner products involve communication between processors. On the other hand, Chebyshev acceleration, though not requiring inner products, does require the use of one or more iteration parameters which are related to the largest and smallest eigenvalues of the iteration matrix. 
Procedures for finding those parameters ¿se relatively elementary methods to estimate the extreme eigenvalues of the iteration matrix.

We propose to investigate the use of more sophisticated procedures for approximately determining extreme eigenvalues and other eigenvalues of the iteration matrix as well as some of the associated eigenvectors. The eigenvalues thus obtained can be used to change the iteration parameters to make the Chebyshev acceleration process converge more rapidly. In addition, the eigenvectors can be used to "purify" the approximate solution. Thus, if an eigenvector of the iteration matrix is known, the approximate solution of the linear system can be modified so that the component of the error with respect to that eigenvector is reduced or eliminated. The elimination of several such eigenvector components can result in a substantial speeding up of the iterative process. The procedure is expected to be particularly effective in the case of singular and nearly singular systems. It should be noted that the parameter estimation and purification procedures do not have to be synchronized with the main iteration procedure since the timing of the parameters changes and of the purification procedures is not critical. Moreover, Ritz values and vectors can be determined based on the set of Krylov vectors generated by the main iterative process and these can be used to carry out simultaneous purifications of the errors. It should also be noted, that eigenvalues and eigenvectors generated in the process of solving a given system can be saved and reused later to speed up the solution of related systems. This should be particularly useful in the solution of time dependent equations by implicit methods and for nonlinear problems solved using nonlinear successive approximation.

\subsection{Parallel Multigrid Methods}

Multigrid methods, when applied appropriately to linear systems arising from elliptic partial differential equations, often converge very rapidly. Frequently the number of cycles required is independent of the grid size $h$. Standard multigrid methods are not completely parallelizable since as one progressively considers coarser and coarser grids fewer points are involved and a point of diminishing parallel return is ultimately reached. Frederickson and McBryan [1991] considered the use of parallel multigrid methods where, at each coarse grid level, several coarse grids are used in parallel so that the total number of grid points used is the same for all levels. The advantages of this class of parallel multigrid methods include the fact that all processors are kept busy at all levels and that the problem of "aliasing", which frequently occurs with standard multigrid methods, is eliminated. Also, the analysis of parallel multigrid methods, at least for some model problems, is much simpler than for standard multigrid methods.

Dr. Vona and Mr. Xiao, together with Dr. Young have worked extensively with Dr. Frederickson on the analysis of the convergence properties of parallel multigrid methods and other multilevel methods in terms of the standard theory of iterative methods. (See Young and Vona [1990] and Vona [1992]). The focus of the work has been on the application of parallel multigrid methods to several types of elliptic problems including anisotropic problems. We are planning to test these algorithms and implement them on distributed memory computers. We also plan to work on the application of the algorithms to more general problems.

\subsection{Time Dependent Problems and Rational Iteration}

We plan to work on the development of iterative methods based on rational iteration for solving the system

$$
\mathrm{A} u=b \text {. }
$$

The motivation for considering rational iterative methods is the fact, that for the time dependent problem 


$$
\left\{\begin{array}{l}
\frac{d u(t)}{d t}=-A u^{\prime}(t)+b \\
u(0)=u^{(0)}
\end{array}\right.
$$

where $A$ is a symmetric and positive matrix, the solution is

$$
u(t)=\bar{u}+e^{-A t}\left(u^{(0)}-\bar{u}\right)
$$

where $\bar{u}=A^{-1} b$ is the solution of (4). Moreover $u(t) \rightarrow \bar{u}$ as $t \rightarrow \infty$. Many of the standard methods for solving the time dependent problem (5) correspond to iterative methods for solving the linear system (4). For example, the extrapolated Richardson method given by $u^{(n+1)}=u^{(n)}+\gamma\left(b-A u^{(n)}\right)$ corresponds to the forward diserence method for integrating (5).

It is well known that, if explicit methods are used to solve (5), then a very severe limitation on the time step $\Delta t$ must be imposed in order to avoid instability. In order to be able to use a larger step size without loss of stability, implicit methods such as the backward difference method or the Crank-Nicolson method are often used. These implicit methods correspond to the following rational iterative method:

$$
(A+\rho I) u^{(n+1)}=[(1-\gamma) A+\rho I] u^{(n)}+\gamma b
$$

Here $\rho$ is a parameter and $\gamma$ is an extrapolation factor. (The choice $\gamma=1$ corresponds to the backward difference method with $\Delta t=\rho^{-1}$, while the choice $\gamma=2$ corresponds to the CrankNicolson method with $\Delta t=2 p^{-1}$ ).

Rational iteration has several potential advantages including the following

(a) by the use of partial fraction decompositions several iterations with different values of $\rho$ can be carried out in parallel. (This was done by Gallopoulos and Saad [1992] for carrying out several time steps in parallel for the time-dependent problem (5)).

(b) by choosing an appropriate set of values $\rho_{1}, \rho_{2}, \ldots \rho_{n}$, (see Young and Vona [1992] )the number of rational iterations required for convergence is $\mathrm{O}(\log K(A))$.

A major obstacle to the use of rational iteration is the need to solve many linear systems of the form $(A+\rho I) x=y$ for some $\rho>0$. This, of course, becomes increasingly difficult as $\rho$ decreases. We plan to investigate several methods for carrying out rational iteration. One procedure is to consider a family of related systems

$$
\left(A+\rho_{i} I\right) x^{(i)}=y^{(i)}
$$

for a set of values $\rho_{1}>\rho_{2}>\ldots>\rho_{m}$. If $\rho_{1}$ is large, then the system can be easily solved by a standard iterative method. The subsequent systems will then be solved by a continuation process which is related to the problem of solving the time-dependent equation. Since the systems to be solved are related, any information, such as eigenvalue/eigenvector estimates obtained for 
solving one system can be saved and used on the next system. We also plan to consider other methods for solving sets of related systems including those described by Van de Vorst [1987] and Saad [1985].

\subsection{Nonsymmetric Systems}

If the matrix $A$ of a given linear system $A u=b$ is symmetric and positive definite (SPD) then many of the standard basic iterative methods such as Richardson's method, the Jacobi method, and the SSOR method are "symmetrizable". This implies, among other things, that the eigenvalues of the iteration matrix $G$ are real and less than one. Because of this, the rate of convergence can be greatly accelerated by the use of Chebyshev acceleration or by conjugate gradient acceleration; see for instance Hageman and Young [1981].

For many practical problems involving convective transport such as the fluid flow and reservoir problems mentioned previously, the linear system $A u=b$ corresponding to the centraldifference finite difference method or Galerkin finite elements is not SPD. For such problems, the standard basic iterative methods are not symmetrizable. However, in many cases the basic iterative method can still be accelerated by the use of generalized conjugate gradient methods (which are often referred to as "Krylov space methods".) Young and Jea [1980] considered three Krylov space methods, namely ORTHODIR, ORTHORES, and ORTHOMIN. The ORTHOMIN form is closely related to the classical form of the conjugate gradient method given by Hestenes and Stiefel [1952] while ORTHORES is a three-term method which was considered by Concus, Golub and O'Leary [1976]. The ORTHODIR form is in theory the most robust since if either of the other two methods converges then ORTHODIR converges. On the other hand ORTHODIR requires somewhat more work per iteration and is subject to rounding errors; see e.g. Abbassian [1983].

A significant disadvantage of Krylov subspace methods is that the amount of work required to carry out a given iteration increases linearly as the number of iteration increases. This can be avoided by using "truncation" or "restarting". However, this may result in slower convergence or failure to converge. Lanczos methods are similar to Krylov space methods but the work required per iteration does not increase with the number of iterations. Jea and Young [1983] considered three Lanczos methods, namely LANDIR, LANMIN, and LANRES. (In Jea and Young [1983] these methods were referred to as "Lanczos/ORTHODIR", "Lanczos/ORTHOMIN", and "Lanczos/ORTHORES," respictively). LANDIR, LANMIN, and L.ANRES correspond to applying ORTHODIR, ORTHOMIN and ORTHORES, respectively, to a "double system" derived from the given system. LANMIN is equivalent to the "biconjugate gradient method" (BCG method) presented by Fletcher [1976]. It can be proved that LANDIR converges whenever either LANMIN or LANRES converge. However, numerical studies indicate that LANDIR suffers from degradation due to roundoff effects.

The BCG method is a widely used method which has been the subject of a considerable amount of research over the past few years. The method is subject to the possibility of breakdown and, even when breakdown does not occur, the convergence behavior is often very irregular. Also, the method requires the use of $A^{T}$ as well as $A$ and this may be a serious disadvantage especially with highly parallel computers. Some of the recent results obtained for BCG are described in the work of Joubert [1990] (for coping with breakdown), Van der Vorst [1992] (for modifying BCG to obtain BCGSTAB which exhibits more regular convergence behavior) and Freund and Nachtigal [1991] (for modifying BCG to obtain QMR which does not require the use of $A^{T}$ ) and still has more regular behavior.

We plan to study the behavior of LANDIR to determine why there are serious roundoff problems and how they can be avoided. We will also study a new procedure which we call "LANGMRES" and which is based on the application of GMRES to the double systems referred to earlier. (GMRES was introduced by Saad and Schultz [1986], and is mathematically equivalent to ORTHODIR but requires less work per iteration and is less subject to rounding errors). 
We also plan to study the use of LANDIR or LANGMRES as auxiliary procedures to be used in case of breakdown for algorithms where BCG or LANRES is used as the primary procedure.

\subsection{Software Research}

The Center for Numerical Analysis has a continuing program of research on iterative algorithms for solving sparse linear systems of algebraic equations with special emphasis on systems arising in the numerical solution of partial differential equations. An important aspect of the work has been the development of several research-oriented software packages developed as part of the ITPACK Project. These packages allow for the use of a wide variety of iterative algorithms suitable for use on vector and parallel supercomputers for both symmetric and nonsymmetric systems.

In the proposed research, we will focus on iterative algorithms from these packages and new extensions of these algorithms that are the best candidates for parallelization. Protorype codes will be implemented on various parallel computers and benchmark performance studies made. The algorithms will also be utilized in large-scale applications.

ITPACKV 2D is a package of seven iterative algorithms for solving sparse linear systems with symmetric and positive definite or mildly nonsymmetric coefficients matrices. The iterative algorithms in this package have been implemented for efficient use on vector supercomputers. A parallel version of the package has been developed for use on the 8-processor Cray Y-MP.

NSPCG (Non-Symmetric Preconditioned Conjugate Gradient methods) contains acceleration techniques in conjunction with various preconditioners for solving large sparse nonsymmetric linear systems by iterative algorithms. This package is modular in nature so that almost any preconditioner may be used with any accelerator.

Part of the research involves the problem of extending this software capability to parallel machines. We also plan to consider parallel variants of recent stabilized schemes such as the Quasi-minimum Residual (QMR) method and Stabilized Biconjugate Gradient (BCGSTAB).

The software will be applied to convective transport applications in 3D.

Recent work here and elsewhere has focused on the establishment of a set of computational kernels related to iterative methods for solving sparse linear systems. It can be shown that many iterative algorithms can be decomposed into a relatively small set of basic computational operations. These computational kernels provide a flexible platform and would be particularly advantageous for software developed for use on different advanced parallel architectures. Since these would be the most computationally intensive parts of an iterative algorithm, it should be possible to develop efficient iterative algorithms for different parallel computers by writing code in terms of these basic building blocks. Like the original Basic Linear Algebra Subprograms (BLAS), these computational kernels would be defined in terms of fundamental operations independent of the computer architecture and without giving the exact implementation details for the particular underlying parallel computer architecture. Obviously, different hardware characteristics (shared versus distributed memory) would require different programming strategies to achieve optimal performance. Also, an iterative code could use the computational kernels tailored for a particular parallel computer and thereby be optimized in some sense. Moreover, the same code would be portable to other parallel computers having a similar set of optimized computational kernels.

During the development of the NSPCG software package, computational kernels were used related to vectorization. These were the key routines in the iterative algorithms that were optimized for efficient execution on vector computers. Several different sparse matrix data storage schemes were available in NSPCG for representing matrices ranging from unstructured to diagonally structured. By writing the iterative algorithms in terms of computational kernels, basic operations were vectorized using these storage schemes. While these computational kernels proved to be quite useful on vector computers, it is unclear if the same set of kernels or a different one will be need on parallel computers.

The development of these parallelizable computational kernels are complicated by the various sparse matrix storage formats to be treated and the different parallel computer 
architectures involved. The communication overhead is also an issue to be investigated. Part of the research involves developing a taxonomy of iterative kernels and analyzing their computational complexity. Then representative parallel kernels will need to be developed and tested in PDE applications.

As an example of what we have in mind, some of the "Iterative BLAS" from Oppe and Kincaid [1990] are presented next. The Iterative BLAS are organized into three groups: general vector update operations, linear recursions, and stencil-specific vector update operations. We give the first two here.

We define the following General Vector Update Operations routines. Let $x, y$, and $a$ be real vectors, $\alpha$ be a real scalar, and $m$ and $n$ be integer vectors.

YAX ( $y$ plus $a$ times $x) y_{i} \leftarrow y_{i}+\alpha a_{i} x_{i}$.

YASX ( $y$ plus $a$ times sparse $x) y_{i} \leftarrow y_{i}+\alpha a_{i} x_{m i}$.

SYAX (sparse $y$ plus $a$ times $x$ ) $y_{n i} \leftarrow y_{n i}+\alpha a_{i} x_{i}$.

SYASX (sparse $y$ plus $a$ times sparse $x$ ) $y_{n i} \leftarrow y_{n i}+\alpha a_{i} x_{m i}$.

YAX2 (y plus $a$ times $x$, Level 2) $y_{i} \leftarrow y_{i}+\alpha \sum_{j=1}^{k} a_{i, j} x_{i+m}$.

YASX2 ( $y$ plus $a$ times sparse $x$, Level 2) $y_{i} \leftarrow y_{i}+\alpha \sum_{j=1}^{k} a_{i, j} x_{m i, j}$.

We define the following Linear Recursion routines. Let $\alpha$ be a real scalar and $x, a, b$, and $d$ be real vectors.

FOLR (first order linear recursion) $x_{i} \leftarrow x_{i}+\alpha a_{i} x_{i-1}$.

SOLR (second order linear recursion) $x_{i} \leftarrow x_{i}+\alpha\left(a_{i} x_{i-1}+b_{i} x_{i-2}\right)$.

MFOLR (multiple first order linear recursion) $x_{i, j} \leftarrow x_{i, j}+\alpha a_{i, j} x_{i-1, j}$.

MSOLR (multiple second order linear recursion) $x_{i, j} \leftarrow x_{i, j}+\alpha\left(a_{i, j} x_{i-1, j}+b_{i, j} x_{i-2, j}\right)$.

One of our goals is to develop and test software for computational kernels that are common to a large class of iterative methods. These kernels can then be used in the development and testing of software for large systems arising from applications. Several different researchers have begun to write software for computational kernels to be used in developing efficient and portable implementations of iterative algorithms for sparse matrix problems on high performance computers. Some of the problems that have to be overcome concern keeping the interface for the suite of codes simple yet functional and flexible for many different data structures and for various iterative algorithms. This is particularly difficult to achieve on different parallel architectures.

Some related work for iterative software is summarized below:

Ashby and Seager [1990] propose a standard user interface for iterative linear system solvers. They discuss how the matrix-vector multiplication, preconditioning, and stopping criterion routines might be standardized as well as the overall design of iterative packages. Their goal is to make it easier for users to integrate, experiment,and combine iterative software into scientific simulation codes.

Saad [1991] describes the main features of a toolkit for manipulating and working with sparse matrices. He states that one of the goals of the package is to provide basic tools to facilitate the exchange of software and data between researchers in sparse matrix computations. 
The package provides programs for converting data structures, printing simple statistics for a matrix, plotting a matrix profile, performing basic linear algebra operations with sparse matrices, etc. Recently, a version has been developed for use on a workstation with color graphics.

Oppe [1992] is developing a software package call STENCIL to solve linear systems by various iterative methods. The package contains several acceleration methods in conjunction with a number of preconditioners. The coefficient matrix is assumed to arise from the use of certain stencils in either a 2-D rectangular or 3-D box domain.

Joubert and Carey [PCG] are developing a package called PCG designed to solve linear systems by iterative methods on a variety of parallel architectures. Uniformity of calling sequences and near-uniformity of matrix storage formats are maintained across shared memory, data parallel, and message passing computers.

Heroux [1992] presents a proposal for a toolkit of kernel routines for some of the basic operations in iterative methods for sparse linear systems. He describes an interface for routines that perform the product of a sparse matrix times a dense matrix, the solution of a sparse triangular system with multiple right-hand-sides, the right permutation of a sparse matrix, and a check for the integrity of a sparse matrix representation. The interfaces for these four operations are defined for a variety of common data structures and a set of guidelines is given for defining the interfaces for new data structures. Heroux indicates that the primary purpose of his toolkit is to provide a set of basic routines upon which Duff, Marrone, and Radicati [1992] can build their user level sparse BLAS.

Duff, Marrone, and Radicati [1992] describe a set of level 3 basic algebra subprograms for sparse matrices. They discuss the design, implementation, and use of routines for the multiplication of a full matrix by a sparse matrix, for the solution of triangular systems with one or more (full) right-hand sides, for transforming sparse data structures, and for permuting sparse and full matrices. The objective is to develop standard software for use in the development of efficient and portable codes using iterative algorithms with sparse matrices on high performance computers. Their work is intended to be complementary to the toolkit of Heroux [1992].

Barrett, Berry, Demmel, Dongarra, Eijkhout, and Pozo [1992] describe a software system based on templates and tool boxes designed to facilitate the preparation of programs for implementing iterative algorithms for solving large sparse linear systems. The main component of the environment is a sparse matrix package for handling operations involving sparse matrices and vectors. The use of the system is illustrated for a number of iterative algorithms. The algorithms are described in three ways: first using an Algol-like language; second using a Matlab implementation; and third using Fortran. Extensive use is made of subroutines for carrying out various operations involving matrices and vectors. One of the goals is to be able to write a new program and to modify an existing program for describing an iterative algorithm using a relatively small number of statements.

Each of theses approaches has considerable merit but there is no clear consensus in this active research area. Our proposed work on iterative kernels will complement these activities. Hence we anticipate continuing collaboration with several of the above researchers on this subject.

\subsection{Applications}

Navier Stokes problems and reservoir simulation are two of the main applications areas to be considered in the reserach. For brevity we will give here only a brief description of the reservoir problem. Further details are given in the references cited and in the Appendix.

Numerical reservoir simulation involves the development and usage of a mathematical model and simulator which describes the flow of fluids in a permeable medium. The most complex simulators are those used for enhanced oil recovery processes. These processes involve the injection of chemicals, solvents, or heat into underground reservoirs in order to increase the recovery of trapped oil from the reservoir rock. Processes using chemicals such as detergents, caustics, and polymers are called chemical flooding and typically involve the multiphase flow of a large number of components that interact with the permeable medium. This can be represented 
by a mathematical formulation that involves a set of coupled, highly nonlinear, time-dependent partial differential equations that must be solved by using numerical methods such as finitedifference or finite-element methods. This leads to a system of algebraic equations that must be solved at each time level using a numerical technique.

Due to the complexity of the governing nonlinear chemical flooding equations, both a fine mesh and very small time steps are needed to obtain an accurate solution (Datta Gupta et al., 1986; Pope et al., 1990; and Saad, 1989). Thus, for field simulations, a very large amount of computer time, as well as a large amount of storage, is required. As a consequence, supercomputers are needed to solve such enhanced oil recovery simulation problems. Even then, a highly optimized vector-parallel code is essential for practical and affordable simulation.

Over the past several years, we have developed two major compositional reservoir simulators (Chang et al., 1990; Datta Gupta et al., 1986; Saad et al., 1989; and Saad et al., 1990). One is for simulating enhanced oil recovery by chemical methods and is called UTCHEM. The second is for simulating enhanced oil recovery by miscible agents such as solvents, enriched gases, and carbon dioxide and is called UTCOMP. Both are compositional simulators, by which we mean that compositional phenomena such as phase behavior are modeled. These simulators employ finite-difference algorithms that discretize the nonlinear partial differential conservation equations describing the pertinent chemical and physical phenomena in three dimensions.

We, and others in industry use these simulators for research into process inechanisms, reservoir studies, interpretation of coreflood experiments, evaluation of numerical methods and solution algorithms, and optimization of oil recovery methods, among other uses. Versions of our codes have been developed for Cray computers and VAX computers. The Cray version of UTCHEM is highly vectorized and executes on a CRAY Y-MP 8 using Multitasking ${ }^{\mathrm{TM}}$. UTCOMP has only been partially vectorized, so it does not execute nearly as efficiently as UTCHEM. In both cases, there are many problems that require very large computational times and storage. Single-well patterns can be simulated in most cases without excessive cost, but as an application expands to include many patterns or an entire field of even moderate size, the cost often becomes prohibitive. Even laboratory-scale simulations can be very expensive when highresolution simulation of unstable displacements and/or displacements with larg: crossflow are needed for fundamental investigations. It follows that high performance parallel schemes are needed. A brief description of the UTCHEM simulator and governing equations is provided in Appendix I.

As indicated above, the discretization of the governing partial differential equations describing fluid flow through a permeable medium leads to systems of linear equations. The numerical solution of the resulting systems of equations is very time consuming, especially as the number of equations becomes large. Furthermore, an actual field simulation may require solving these systems thousands of times. It is clear that a significant portion of the computational cost of a reservoir simulation study is incurred by the solution of the linear systems of equations. Hence, the development of efficient solvers is an important aspect of reservoir simulation.

As a part of our ongoing effort on improving our computational techniques for reservoir simulation, we are working on the development of a fully implicit polymer flooding simulator. In this simulator, the governing component conservation and overall material balance (pressure) equations are solved implicitly using a higher-order finite-difference method. The new simulator has improved stability properties over those of the UTCHEM simulator using an IMPES-like formulation. However, the solution of the resulting systems of linear equations is much more complicated and time consuming than the linear systems obtained in UTCHEM. Figure 1 shows the representative matrix structure for a two-dimensional problem with a very coarse grid having $N_{x}=6$ and $N_{y}=6$. The matrix has a large and dense bandwidth and although the structure is symmetric the entries are not. The bandwidth becomes larger as additional components and gridblocks are used (bandwidth $=12 \mathrm{~N}_{\mathrm{X}}+\mathrm{N}_{\mathrm{c}}$, where $\mathrm{N}_{\mathrm{c}}$ is the number of components used in the problem). Fast and robust system solvers are crucial for practical usage of this simulator since over $90 \%$ of the computation time is spent in this portion of the simulator. Our preliminary results with NSPCG show that some nonsymmetric iterative solvers can outperform band solvers. 
Part of the proposed research concerns the development and application of higher order nonoscillating schemes for this class of transport problem. Another important goal is the efficient solution of the resulting sparse systems using iterative methods. Finally, the problem of developing an effective, scalable parallel implementations will be a focal point of the research. 


\subsection{REFERENCES}

Abbassian, R.O., "Lanczos Algorithms for the Acceleration of Nonsymmetrizable Iterative Methods" Report CNA-193, Center for Numerical Analysis, The University of Texas, Austin, Texas, 1983.

Adams, Loyce M., LeVeque, Randall J. and Young, David M., "Analysis of the SOR Method for the 9-point Laplacian," SIAM Journal of Numerical Analysis 25, 1156-1180, 1988.

Ashby, S.F.and M.K. Seager, "A Proposed Standard for Iterative Linear Solvers, Version 1.0," Report UCRL-102860, Lawrence Livermore National Laboratory, January 1990.

Ashcraft, C. "A Moving Computation Front Approach for Vectorizing ICCG Calculations." General Motors Research Publication, GMR-5174, 1985.

Ashcraft, C.and R. Grimes. "On Vectorizing Incomplete Factorizations and SSOR Preconditioners." SIAM Journal on Scientific and Statistical Computing, Vol. 9, No. 1, pp. 122-151, January 1988.

Ashcraft, C.M. Shook, and J. Jones. "A Computational Survey of Conjugate Gradient Preconditioners on the Cray 1-S." General Motors Research Publication, GMR-5299, 1986.

Axelsson, O., and P. Vassilevski, "Algebraic Multilevel Preconditioning Methods II," Report 1988-15, Institute for Scientific Computation, University of Wyoming, Laramie, Wyoming, 1988.

Barragy, E. and G. F. Carey, "A Parallel Element-by-Element Solution Scheme," Int. J. Num. Meth. Eng., 26, 2367-2382, 1988.

Barragy, E. and G. F. Carey, Stream Function Vorticity Solution Using High Degree (p) Finite Elements and Element-by-Element Techniques, Communications in Applied Numerical Methods, In press, 1992.

Barragy, E., G. F. Carey and R. Van de Geijn, Performance and Scalability of Finite Element Analysis for Distributed Parallel Computation, J. of Parallel and Distributed Computing, In Press 1992.

Barragy, E., G. F. Carey and R. Walters, Application of Conjugate Gradient Methods to Tidal Simulation, Advances in Water Resources, Submitted 1992.

Barrett, R.M. Berry, J. Demmel, J. Dongarra, V. Eijkhout, and R. Pozo, "LAPACK Working Note: Building Blocks for Iterative Solution of Linear Systems," Draft Report, Dept. of Computer Science, University of Tennessee, Knoxville, TN, October 1992.

Bhuyan, D., L.W. Lake, and G.A. Pope: "Mathematical Modeling of High-pH Chemical Flooding," SPE Reser. Eng. 5 (2), May 1990.

Carey, Graham F., Kincaid, David R., Sepehrnoori, Kamy, and Young, David M., "Vector and Parallel Iterative Solution of Large Sparse Systems for PDEs", Science and Engineering on

Cray Supercomputers, Cray Research, Inc., Minneapolis, Minn., 25-43, 1989.

Carey, G. F. and E. Barragy, Parallel-Vector Computation with High-p Element-by-Element Methods, International J. of Computer Mathematics, In press, 1991.

Carey, G.F. and S. Bova, "Parallel Grid Generation/Optimization Using Block Domain Decomposition," Journal of Computational Physics, 8, 243-256, 1992.

Carey, G. F. and T. T. Pan, Shocked Transonic Flow Calculations Using Finite Elements and a Fictitious Gas,Computer Methods in Applied Mechanics and Engineering, 81, 1990.

Carey, G., F., J. Schmidt, V. Singh and D. Yelton, A Scalable, Object-Oriented Finite Element Solver for Partial Differential Equations on Multicomputers, J. of Parallel and Distributed Computing, Submitted 1992.

Carey, G. F., Grid Generation and Optimization, Wiley, (in preparation), 1993.

Carey, G. F., "Parallel Sub-Domain and Element-by-Element Techniques," in Parallel Supercomputing, Wiley, Chichester, 1989.

Carey, G. F., E. Barragy, R. McLay, and M. Sharma, "Element-by-Element Vector and Parallel Computations, " $C A N M, 4,3,299-307,1988$.

Chang, Y., G.A. Pope, and K. Sepehmoori: "A Higher-Order Finite Difference Compositional Simulator," J. Pet. Sci. Eng. 5 (1), November 1990. 
Concus, P., G.H. Golub, and D.P. O'Leary, "A Generalized Conjugate Gradient Method for the Numerical Solution of Elliptic Partial Differential Equations", appeared in Sparse Matrix Computation (J.R. Bunch and D.J. Rose, eds.), Academic Press, New York, 1976.

Datta Gupta, A., G.A. Pope, K. Sepehrnoori, and R.L. Thrasher: "A Symmetric, Positive Definite Formulation of a Three-Dimensional Micellar/Polymer Simulator," SPE Reser. Eng. 1 (4), November 1986.

Davis, M. and G. F. Carey, Iterative Solution of the Stream Function-Vorticity Equations Using a Multigrid Solver with Finite Elements, Communications in Applied Numerical Methods, In press 1992.

Dayde, M. J.and I. S. Duff. "Use of Level 3 BLAS in LU Factorization on the Cray-2, the ETA10P, and the IBM 3090-200/Vr." Harwell Laboratory, Report CSS 229, October 1988.

Demmel, J.J., J. Dongarra, J. DuCroz, A. Greenbaum, S. Hammarling, and D. Sorenson. "Prospectus for the Development of a Linear Algebra Library for High-Performance Computers." Argonne National Laboratory, Technical Memorandum No. 97, September 1987.

Dodson, D. S. and J. G. Lewis. "Issues Relating to Extension of the Basic Linear Algebra Subprograms." ACM SIGNUM Newsletter, Vol. 20, No. 1, pp. 19-22, January 1985.

Dodson, D. S.and J. G. Lewis. "Proposed Sparse Extensions to the Basic Linear Algebra Subprograms." ACM SIGNUM Newsletter, Vol. 20, No. 1, pp. 22-25, January 1985.

Dodson, D., S.R. G. Grimes, and J. G. Lewis. "Sparse Extensions to the FORTRAN Basic Linear Algebra Subprograms." To be published in ACM Transactions on Mathematical Software.

Dodson, D. S.R. G. Grimes, and J. G. Lewis. "Model Implementation and Test Package for the Sparse Basic Linear Algebra Subprograms." To be published in ACM Transactions on Mathematical Software.

Dongarra, J. J., J. R. Bunch, C. B. Moler, and G. W. Stewart. LINPACK Users' Guide. Philadelphia: SIAM Publications, 1979.

Dongarra, J. J., J. DuCroz, I. Duff, and S. Hammarling. "A Set of Level 3 Basic Linear Algebra Subprograms." Argonne National Laboratory, ANL-MCS-TM 88 (Revision 1), May 1988.

Dongarra, J. J.,J . DuCroz, I. Duff, and S. Hammarling. "A Set of Level 3 Basic Linear Algebra Subprograms: Model Implementation and Test Programs." Argonne National Laboratory, ANL-MCS-TM 119 (Revision 1), June 1988.

Dongarra, J. J., J. DuCroz, S. Hammarling, and R. Hanson. “An Extended Set of Fortran Basic Linear Algebra Subprograms." ACM Transactions on Mathematical Software, Vol. 14, No. 1, March 1988.

Dongarra, J. J., J. DuCroz, S. Hammarling, and R. Hanson. "An Extended Set of Fortran Basic Linear Algebra Subprograms: Model Implementation and Test Programs." ACM Transactions on Mathematical Software, Vol. 14, No. 1, pp. 18-32, March 1988.

Dongarra, J. J., J. DuCroz, S. Hammarling, and R. Hanson. "A Proposal for an Extended Set of Fortran Basic Linear Algebra Subprograms." ACM SIGNUM Newsletter, Vol. 20, No. 1, pp. 2-18, January 1985.

Dongarra, J. J. and S. C. Eisenstat. "Squeezing the Most Out of an Algorithm in CRAY Fortran." ACM Transactions on Mathematical Software, Vol. 10, No. 3, pp. 219-230, September 1984.

Duff, I.M., M. Marrone, and G. Radicati, “A Proposal for User Level Sparse BLAS,” Rutherford Appleton Laboratory, Oxfordshire, England, or CERFACS, Toulouse, France, or IBM Semea, Cagliari, Italy, September 1992.

Fischer, P.F., Parallel Spectral element Methods for the Incompressible Navier-Stokes Equations, Ph.D. Thesis, Massachusetts Institute of Technology, in progress, 1989.

Fletcher, R., "Conjugate Gradient Methods for Indefinite Systems", Lecture Notes in Mathematics 506, Springer-Verlag, New York, 1976.

Frederickson, P.O. and O.A. McBryan, "Recent Developments for the PSMG Multiscale Method," Report CU-CS-524-90, Department of Computer Science, University of Colorado at Boulder, CO, 1991. 
Freund, Roland W. and N. Nachtigal, "QMR: a Quasi-Minimal Residual Method for NonHermitian Linear Systems," Numerische Mathematik, 60, 315-339, 1991.

Gallivan, K., W. Jalby, U. Meier, and A. Sameh. "The Impact of Hierarchical Memory Systems on Linear Algebra Algorithm Design." University of Illinois at Urbana-Champaign, Center for Supercomputing Research and Development. Report CSRD 625, September 1987.

Gallopoulos, E. and Y. Saad, [1992]. "Efficient Solution of Parabolic Equations by Krylov Approximation Methods" SIAM J. Sci. Statist. Comput. 13, 1236-1264.

Garbow, B. S., J. M. Boyle, J. J. Dongarra, and C. B. Moler. Matrix Eigensystem RoutinesEISPACK Guide Extension. Springer-Verlag, Lecture Notes in Computer Science 51, 1977.

Glowinski, R., G.H. Golub, G.A. Meurant and J. Periaux (eds.), Domain Decomposition Methods for Partial Differential Equations, SIAM, 1988.

Gustafson, J., G.R. Montry, and R.E. Benner, "Developrnent of Parallel Methods for a 1024Processor Hypercube," SIAM J. Sci. Stat. Comput., 9,4, 609-638, 1988.

Hageman, L.A. and D.M. Young: Applied Iterative Methods, Academic Press, Inc., New York, 1981.

Heroux, M.A. “A Proposal for A Sparse BLAS Toolkit,” Cray Research, Inc., Eagan, MN, May 1992.

Hestenes, M.R. and E.L. Stiefel, "Methods of Conjugate Gradients for Solving Linear Systems" Nat. Bur Std. Res. 49, 409-436, 1952.

Jea, Kang C. and D.M. Young, "On the Simplification of Generalized Conjugate-1 'radient Methods for Nonsymmetrizable Linear Systems", Linear Algebra and Its Applications, 52/53, 399-417, 1983.

Jordan, T. L. "A Guide to Parallel Computation and Some Cray-1 Experiences." Parallel Computations (G. H. Rodrigue, ed.), pp. 1-50. New York: Academic Press, 1982.

Joubert, W.D. and G.F. Carey. "PCG User's Manual: A Package for the Iterative Solutions of Large Sparse Linear Systems on Scalar, Vector and Parallel Computers," Los Alamos National Laboratory and The University of Texas at Austin, draft report.

Joubert, Wayne D., "Lanczos Methods for the Solution of Nonsymmetric Systems of Linear Equations," to appear in SIAM Journal of Matrix Analysis and Applications., 1992.

Joubert, Wayne D., and Carey, Graham F., "Parallelizable Restarted Iterative Methods for Nonsymmetric Linear Systems. Part I: Theory, Part II: Parallel Implementation," May 1991, to appear in International Journal of Computer Mathematics, Gordon Breach publ., special issue on preconditioned conjugate gradient methods; also Report CNA-251, The University of Texas at Austin, May 1991.

Joubert, Wayne D., Highman, Peter, and Carey, Graham F., "PCG/CM: A Package for the Iterative Solution of Large Sparse Linear Systems on the Connection Machine," to appear in the Proceedings on Parallel Processing for Scientific Computing, Houston, Texas, 1991.

Joubert, Wayne D., and Manteuffel, Thomas A., "Iterative Methods for Nonsymmetric Linear Systems," appeared in Iterative Methods for Linear Systems, (David R. Kincaid and Linda J. Hayes, eds.), Academic Press, Boston, 149-171, 1991.

Joubert, Wayne D., Manteuffel, Thomas A., Parter, Seymour V., and Wong, Sze-Ping, "Preconditioning Second-Order Elliptic Operators: Experiment and Theory," Proceedings of Copper Mountain Conference on Iterative Methods, April 1990; SIAM Journal of Scientific and Statistical Computation., 13, 1, 259-288, 1992.

Joubert, Wayne, and Oppe, Thomas C., "Improved SSOR and Incomplete Cholesky Solution of Linear Equations on Shared Memory and Distributed Memory Parallel Computers," Report CNA-253, Center for Numerical Analysis, University of Texas at Austin, 1991.

Joubert, Wayne. "On the Convergence Behavior of the Restarted GMRES Algorithm for Solving Nonsymmetric Linear Systems," Submitted January 1992 to Journal of Numerical Linear Algebra with Applications, special issue on Iterative Methods and Preconditioners for Nonsymmetric Linear Systems.

Joubert, Wayne. "A Robust GMRES-Based Adaptive Polynomial Preconditioning Algorithm for Nonsymmetric Linear Systems," To be Submitted to SIAM Journal on Matrix Analysis and 
Applications, Special issue for Second Copper Mountain Conference on Iterative Methods, May 1992.

Joubert, Wayne, and Graham F. Carey. "Embedded Iterative Solution of Nonlinear PDE's on the Connection Machine," International Journal for High Speed Computing, Submitted 1992.

Joubert, W., "Iterative Methods for the Solution of Nonsymmetric Systems of Linear Equations", Report CNA-242, Center for Numerical Analysis, The University of Texas, Austin, Texas, 1990.

Kang, L., Domain Decomposition and Parallel Algorithms, Wuhan University Press, Wuhan University, Peoples Republic of China, 1987.

Kincaid, D.R., Carey, G.F., Oppe, T.C., Sepehrnoori, K., and Young, D.M., "Combining Finite Element and Iterative Methods for Solving Partial Differential Equations on Advanced Computer Architectures," Advances in Computer Methods for Partial Differential Equations V (R. Vichnevetsky and R.S. Stepleman, eds.), publ. IMACS, Dept. of Computer Science, Rutgers University, New Brunswick, N.J., 375-378, 1984.

Kincaid, D.R., Oppe, T.C., Respess, J.R. and Young, D.M., "Chapter 7: ITPACK Solution Modules," Solving Elliptic Problems Using ELLPACK (John R. Rice and Ronald F. Boisvert, eds.), Springer-Verlag, 237-258, 1985.

Kincaid, D. R., T. C. Oppe, and D. M. Young. "ITPACKV 2D User's Guide." Report CNA-232, Center for Numerical Analysis, University of Texas at Austin, May 1989.

Kincaid, D. R., J. R. Respess, D. M. Young, and R. G. Grimes. "ITPACK 2C: A FORTRAN Package for Solving Large Sparse Linear Systems by Adaptive Accelerated Iterative Methods." ACM Transactions on Mathematical Software, Vol. 8, No. 3, pp. 302-322, September 1982.

Kincaid, David R. and Young, David M., "A Brief Review of the ITPACK Project," Journal of Computational and Applied Mathematics 24, 121-127, 1988.

Kincaid, David R. and Oppe, Thomas C. Recent vectorization and parallelization of ITPACK. In O. Axelsson and L.Y. Kolotilina, editors, Preconditioned Conjugate Gradient Methods, Lecture Notes in Mathematics 1457, pages 58-78. Springer-Verlag, New York, 1990. .

Kincaid, D.R. and Oppe, T.C., "Recent Vectorization and Parallelization of ITPACK", Preconditioned Conjugate Gradient Methods, pp. 58-78, (O. Axelsson and L.Y. Kolotilnia, eds.), Lecture Notes in Mathematics 1457, Springer-Verlag, New York, NY, 1991.

Kincaid, David R., Oppe, Thomas C. and Joubert, Wayne D., "An Introduction to the NSPCG Package", International Journal for Numerical Methods in Engineering, 27, 589-608, 1989.

Lawson, C. L.,R. J. Hanson, D. R. Kincaid, and F. T. Krogh. "Basic Linear Algebra Subprograms for Fortran Usage." ACM Transactions on Mathematical Software, Vol. 5, No. 3, pp. 308-323, September 1979.

MacKinnon, R., G. F. Carey, and P. Murray, A Procedure for Calculating Vorticity Boundary Conditions in the Stream Function-Vorticity Method, Communications in Applied Numerical Methods, 6, 1990.

MacKinnon, R. and G. F. Carey, Moving-Grid Finite Element Modelling of Thermal Ablation and Consolidation in Porous Media, International Journal for Numerical Methods in Engineering, In press 1992.

Madsen, N. K., G. H. Rodrigue, and J. I. Karush. "Matrix Multiplication by Diagonals on a Vector/Parallel Processor." Information Processing Letters, Vol. 5, No. 2, pp. 41-45, June 1976.

McBryan, O., "New Architecutres: Performance Highlights and New Algorithms," Parallel Computing, 7, 477-499, 1988.

Oppe, T., W. Joubert, and D. Kincaid: "NSPCG User's Guide Version 1.0," Center for Numerical Analysis, CNA-217, U. of Texas, Austin, April 1988.

Oppe, T. C. The Iterative Solution of Large Sparse Linear Systems Using Vector Computers. Doctoral dissertation, University of Texas at Austin, May 1990.

Oppe, T.C. "STENCIL User's Guide: A Package for Solving Sparse Linear Systems by Various Iterative Methods," Supercomputer Computations Research Institute, Florida State University, December 1992. 
Oppe, T. C., W. D. Joubert, and D. R. Kincaid. "NSPCG User's Guide, Version 1.0: A Package for Solving Large Sparse Linear Systems by Various Iterative Methods." Report CNA-216, Center for Numerical Analysis, University of Texas at Austin, April 1988.

Oppe, T.C. and D.R. Kincaid, “Are There Iterative BLAS?" Report CNA-240, Center for Numerical Analysis, University of Texas at Austin, February 1990.

Ortega, J.M., Introduction to Parallel and Vector Solution of Linear Systems, Plenum Press, New York, 1988.

Pope, G.A., L.W. Lake, and K. Sepehrnoori: "Modeling and Scale-up of Chemical Flooding: Third and Final Annual Report for the Period October 1987-September 1988," prepared for the U.S. Department of Energy under Contract No. DE-AC19-85BC10846, Bartlesville, Oklahoma, 1990.

Ramdas, Malathi, and Kincaid, David R. "Parallelizing ITPACKV 2D for the Cray Y-MP." In R. Beauwens and R. De Groen, editors, Iterative Methods in Linear Algebra, pages 323-337. Elsevier Science Publishers B.V. (North-Holland), New York, 1992.

Ramdas, Malathi, and Kincaid, David R. [1991]. "Parallelizing ITPACKV 2D." In R. Vichnevetsky and J.J.H. Miller, editors, IMACS '91, volume 2, pages 686-687. Criterion Press, Dublin, 1991.

Saad, N. Field Scale Simulation of Chemical Flooding, Ph.D. dissertation, U. of Texas, Austin, 1989.

Saad, Y., "On the Lanczos Method for Solving Symmetric Linear Systems with Several Righthand Sides" Tech Report YALEU/DCS/R R-396, Department of Computer Science, Yale University, New Haven, CT, 1985.

Saad, N., G.A. Pope, and K. Sepehrnoori: "Application of Higher-Order Methods in Compositional Simulation," SPE Reser. Eng. 5 (1), November 1990.

Saad, Y. "SPARSKIT: A Basic Tool Kit for Sparse Matrix Computations," RIACS Technical Report 90.20, NASA Ames Research Center, May 1990. (Updated January 1991, University of Minnesota, Minnerpolis, MN).

Saad, Y. and M. Schultz, "GMRES: a Generalized Minimum Residual Algorithm for Solving Nonsymmetric Linear Systems," SIAM J. Sci. Stat. Comput. 7, 856-869, 1986.

Santiago, Marilyn and Kincaid, David R. "Using Cyclic Reduction on a Parallel Computer to Improve the Performance of an Underwater Sound Implicit Finite Difference Model," Computers in Mathematical Applications, 21(5): 83-94, 1991.

Seager, M. and G. F. Carey, Adaptive Domain Extension and Adaptive Grids for Unbounded Spherically Elliptic PDE's, SIAM Journal of Scientific and Statistical Computing, Vol. 11, No. 1, 92-111, 1990.

Schreiber, R., Block Algorithms for Parallel Machines, Technical Report 87-5, Rensellaer Polytechnic Institute, Department of Computer Science, 1987.

Shadid, J.N. and Tuminaro, R.S., "A Comparison of Preconditioned Nonsymmetric Krylov Methods on a Large-Scale MIMD Machine," Sandia National Laboratories Report, SAND 91-0333, Albuquerque, NM, 1991.

Shadid, J.N., and Tuminaro, R.S., "Iterative Methods for NonsymmetricSystems on MIMD Machines," Proceedings of the Fifth SIAM Conference on Parallel Processing for Scientific Computing, Houston, Texas, March 25-27, 1991.

Shadid, J.N. and Tuminaro, R.S., "Sparse Iterative Algorithm Software for Large-Scale MIMD Machines: An Initial Discussion and Implementation," Sandia National Laboratories Report, SAND 91-0059,Albuquerque, NM, January, 1991.

Smith, B. T., J. M. Boyle, J. J. Dongarra, B. S. Garbow, Y. Ikebe, V. C. Klema, and C. B. Moler. Matrix Eigensystem Routines-EISPACK Guide, Second Edition Springer-Verlag, Lecture Notes in Computer Science 6, 1976.

Sweet, R., "A Parallel and Vector Variant of the Cyclic Reduction Algorithm," SIAM J. of Sci. Stat. Comp., 9, 761-765, 1988.

Van der Vorst, H. A. "(M)ICCG for 2D Problems on Vectorcomputers." Report No. A-17, Data Processing Center, Kyoto University, Kyoto, Japan, December 1986. 
Van der Vorst, H.A., "Bi-CGSTAB: A Fast and Smoothly Converging Variant of Bi-CG for the Solution of Nonsymmetric Linear Systems" SIAM J. Sci, Stat. Comp. 13, 631-644, 1992.

Vona, B.R., "Parallel Multilevel Iterative Methods" Report CNA-261, Center for Numerical Analysis, The University of Texas at Austin, Austin, Texas, 1992.

Wu, H. and G. F. Carey, Non-Linear Convective Effects on Moving Boundary AC Plasma Arcs, IEEE. Transactions on Plasma Science, In Press, 1992.

Young, David M. and K.C. Jea, K.C., "Generalized Conjugate Gradient Acceleration of Nonsymnictrizable Iterative Methods", Linear Algebra and Its Applications 34, 159-194, 1980.

Young, David M. , "Iterative Solution of Large Linear Systems" Academic Press, New York, 1971.

Young, David M., "The Search for 'High Level' Parallelism for Iterative Sparse Linear System Solvers," Chapter 7 of Parallel Supercomputing: Methods, Algorithms and Applications, edited by Graham F. Carey, John Wiley and Sons, Ltd, 1989.

Young, David M., Jea, Kang C. and Mai, Tsun-zee, "Preconditioned Conjugate Gradient Algorithms and Software for Solving Large Sparse Linear Systems," appeared in Linear Algebra in Signals, Systems and Control (B.N. Datta, ed.) SIAM, Philadelphia, PA, 1988.

Young, David M. and Vona, Bi Roubolo, "Parallel Multilevel Methods," Report CNA-243, Center for Numerical Analysis, The University of Texas, Austin, Texas, 1990.

Young, David M. and Vona, Bi Roubolo, "On the Use of Rational Iterative Methods for Solving Large Sparse Linear Systems," Applied Numerical Mathematics 10, 261-278, 1992.

Young, David M.,"A Historical Review of Iterative Methods", appeared in A History of Scientific Computing, edited by Stephen G. Nash, History Series, ACM Press, New York, 180-194, 1990.

Young, David M. and Mai, Tsun-zee, "The Search for Omega", Chapter 17 of Iterative Methods for Large Linear Systems, edited by David R. Kincaid and Lincia J. Hayes, Academic Press, Inc., 1990 


\section{Institutional Commitment and Other Support}

The Center for Numerical Analysis is an organized research activity within the College of Natural Sciences. The emphasis of the research is on computer solution of partial differential equations and on the iterative solution of large sparse linear systems. A number of researchoriented software packages have been developed in the Center as part of the ITPACK project to aid in the research.

The CFD Laboratory of the College of Engineering is an endowed Laboratory equipped with several Sun microsystem scientific workstations, $x$-window terminals and Macintosh PC's networked to a Laserwriter. Both the CNA and CFD Labs are linked externally to the U.T. System CRAY YMP via a high-speed line and to national supercomputer centers. An Intel i860 distributed parallel system was obtained through a grant from Intel last year and will be utilized in the research.

We have initiated cooperative efforts with government and industrial researchers in this area. Of particular relevance is the collaboration with the Parallel Processing Institutes and researchers at Sandia and Los Alamos. This association also gives us access to a variety of advanced experimental parallel processors such as the NCUBE and the Connection Machine. Other related cooperative work is underway and industrial support is being requested through fellowships to students working on the project and as summer industrial interns.

\section{Impact on Infrastructure of Science/Engineering}

An important outcome will be not only the algorithm research, but also the software for generic parallel architectures. As in the past, this software will be made available to graduate students and to other researchers. The support will also enhance the academic preparation of graduate students in parallel computing, an area of current need. We hope to move our research forward to lead to major new research contributions in computational modeling on supercomputers. The ITPACK software library is widely distributed and our finite element and grid programs are used in industry and by government research laboratories. (Industrial shortcourses are offered each Fall and Spring on "Computational Aspects of Finite Elements" and include some aspects of high performance computing.) 


\title{
11. BIOGRAPHICAL DATA
}

\author{
Dr. Graham F. Carey
}

Dr. Graham F. Carey is currently a Professor in the Department of Aerospace Engineering and Engineering Mechanics at the University of Texas at Austin. He has a B.S. (Hons.) degree from Australia, and M.S. and Ph.D. degrees from the University of Washington at Seattle. His research and teaching activities primarily deal with techniques in computational mechanics, particularly finite element methods. Related research experience includes periods as a research faculty member in Civil Engineering Australia (1966-68), and as a research engineer at the Boeing Company, Seattle (1968-70), during which time he worked in finite element formulation and computation of nonlinear problems. Prior to joining the University of Texas faculty, he held the positions of Research Assistant to Research Associate Professor at the University of Washington (1974-76), working on computational problems in mechanics. He is now Director of the CFD Laboratory and a member of the Texas Institute for Computational Mechanics, Fluids Group, Center for Nur.erical Analysis and the Center for Enhanced Oil and Gas Recovery Research, where these resea-ch activities are being continued.

Publications include a textbook co-a!nthored with H.C. Martin, entitled Introduction to Finite Element Analysis (McGraw-Hill, 1973), co-authorship of a series of six graduate level monographs entitled Finite Elements (Prentice Hiaii), and co-editorship of the book Finite Elements in Fluids (1985). Dr. Carey has edited a monograph entitled Parallel Supercomputing Methods and Algorithms (Wiley, U.K., 1989) and a monograph on Grid Generation. Redistribution and Refinement is in preparation. He has also authored over 130 publications in the general area of computational mechanics, finite element technology and parallel computing and is editor of the Wiley International Journal, Communications in Applied Numerical Methods.

Additional activities in computational mechanics include membership in the American Academy of Mechanics, SIAM, the Society for Engineering Sciences, Committee on Computational Methods in Engineering Mechanics, with listings in the International Directory of Engineering Analysts, Who's Who in the South and Southwest, Who's Who in Frontiers of Science and Technology, and Who's Who in the World. Dr. Carey is an adjunct fellow of the Minnesota Supercomputer Institute. Dr. Carey has held the W. J. Murray Centennial Teaching Fellowship and has recently received an Engineering Foundation Excellence Award. $\mathrm{He}$ is a recipient of an Endowed Engineering Foundation Professorship and received a high performance computing "Gigaflop" award.

\section{Publications since 1987 (G. F. Carey)}

Books

Parallel Supercomputing: Methods, Algorithms and Applications, John Wiley and Sons, U.K., 1989.

Grid Generation, Redistribution and Refinement, John Wiley \& Sons, U.K., in preparation.

\section{Journal Publications}

Convergence of Iterative Methods in Penalty Finite Element Approximation of the Navier Stokes Equations, J. Comp. Meth. in Applied Mech. and Eng., 60, 1-29, 1987 (with R. Krishnan).

Treatment of Material Discontinuities in Finite Element Computations, IJNME, 24, 393-417, 1987 (with R. MacKinnon). 
Stream Function Solution of Navier-Stokes Problems with Inter-Element Penalties, Int. J. Num. Meth. Fluids, 7, 191-193, 1987, (with M. Utku).

Adaptive Refinement for Least Squares Finite Elements with Element-by-Element Conjugate Gradient Solution, IJNME 24, 569-580, 1987 (with B. N. Jiang).

Nonlinear Preconditioned Conjugate Gradient and Least Squares Finite Elements, Comp. Meth. Appl. Mech. Eng. 62, 145-154, 1987 (with B. N. Jiang).

Least Squares Finite Element Method and Preconditioned Conjugate Gradient Solution, Int. J. Num Meth. Eng. 24, 1283-1286, 1987 (with B. N. Jiang).

Finite Element Error Estimates for Subsonic Flow, J. of Australian Math. Society, Series B 29, 88-102, 1987 (with S. Chow).

Approximate Analysis of Regularized Compressible Flow Using the Fictitious Gas Concept, J. Nonlinear Analysis, Vol. 11, No. 8, 861-875, 1987 (with H. Dinh).

Well Singularities in Reservoir Simulation, J. for the Soc. of Petroleum Engineers, 713-719, November 1987, (with S. Chow).

Moving Elements for Transport Processes, J. for the Soc. of Petroleum Engineers, 401-408, August, 1987, (with A. Mucller, K. Sepehrnoori, and R. Thrasher).

Finite Element Analysis of Mass Transport Through a Viscous Fluid with Reaction, J. of Chem. Eng. Sci., 42, No. 12, 2833-2846, 1987, (with Paul Murray).

Toward Expert Systems in Finite-Element Analysis, CANM, 3, 527-533, 1987, (with P. Patton).

Adaptive Mesh Redistribution for a Boundary Element (Panel) Method, IJNME, 24, 2315-2325, 1987 (with S. Kennon).

Least-Squares Finite Elements for First-Order Hyperbolic Systems, Int. J. Num. Meth. Eng., 26, 81-93, 1988, (with B. N. Jiang).

Finite Element Analysis of Diffusion with Reaction at a Moving Boundary, J. of Comp. Phys., 74, No. 2, 440-455, 1988, (with Paul Murray).

Optimization of Computational Grids, Numerical Grids, Numerical Methods and PDE's, 4, 95117, 1988, (with A. Pardhanani).

Analysis of Material Interface Discontinuities and Superconvergent Fluxes in Finite Difference Theory, J. of Comp. Phys., 75, No. 1, 151-167, 1988 (with R. MacKinnon).

A Stable Least-Squares Finite-Element Method for Nonlinear Hyperbolic Problems, Int. J. Num. Meth. Fluids, 8, 933-942, 1988 (with B. N. Jiang).

Element-by-Element Vector and Parallel Computations, CANM, 4, No. 3, 299-307, 1988 (with E. Barragy, R. McLay and M. Sharma).

A Regularization-Stabilization Technique for Nonlinear Conservation Equation Computations, Int. J. Num Methods PDE's, 4, 105-171, 1988 (with B. Jiang and R. Showalter).

A Partitioning Scheme and Iterntive Solution for Sparse Bordered Systems, Comp. Meth. Appl. Mech. Eng., 70, 321-327, 1988 (with E. Barragy).

Some Aspects of Adaptive Grid Computations, Computers \& Structures, 30, 1/2, 297-302, 1988, (with M. Sharma, K. C. Wang and A. Pardhanani).

A Parallel Element-by-Element Solution Scheme, IJNME, 26, 2367-2382, 1988, (with E. Barragy).

A Class of Data Structures for 2-D and 3-D Adaptive Mesh Refinement, IJNME, 26, 2607-2622, 1988, (with M. Sharma and K. C. Wang).

Extension of Inverse Design Techniques for Multicomponent Airfoils, AIAA Journal, Vol 26, No. 6, 1988, (with M. Siladic).

Performance of Iterative Methods for Newtonian and Generalized Newtonian Flows, IJNMF, 9,127-150, 1989, (with K. Wang and W. Joubert).

Perturbation Analysis of the Shrinking Core Model, J. of Chem. Eng. Sci., Vol. 44, No. 4, $979-$ 983, 1989, (with Paul Murray).

Determination of Interfacial Stress During Thermal Oxidation of Silicon, J. of Applied Physics, 65(9), 3467-3670, May 1989, (with P. Murray).

Superconvergent Derivatives: A Taylor Series Analysis, IJNME, 28, 489-509, 1989, (with R. MacKinnon). 
Coupled Heat Transfer and Viscous Flow and Magnetic Effects in Weld Pool Analysis, UJNMF, 9, 713-730, 1989, (with R. McLay).

Symbolic Eigenvalue Analysis for Adaptive Stepsize Control in PNS Shock Stabilization, Computers and Fluids, 17, No. 4, 527-535, 1989, (with D. Cline).

Semiconductor Device Simulation Using Adaptive Refinement and Flux Upwinding, IEEE Transactions of Computer-Aided Design of Integrated Circuits and Systems, Vol 8, No. 6, 590-598, 1989, (with M. Sharma).

Compressibility Effects in Modeling Thin Silicon Dioxide Films for Semiconductors, J. of the Electrochemical Society, Vol. 136, No. 9, 2666-2673, 1989, (with P. Murray).

Convergence Studies of Least-Squares Finite Elements for First-Order Systems, CANM, Vol 5, 427-434, 1989, (with Y. Shen).

On a Class of Preconditioned Iterative Methods on Parallel Computers, IJNME, Vol 27, No. 3, 637-654, 1989 (with O. Axelsson and G. Lindskog).

Multigrid Solution and Grid Redistribution for Convection-Diffusion, IJNME, Vol. 27, No. 3, 655-664, 1989, (with A. Pardhanani).

A Procedure for Calculating Vorticity Boundary Conditions in the Stream Function-Vorticity Method, CANM, Vol. 6, 47-48, 1990, (with R. MacKinnon and P. Murray).

Adaptive Domain Extension and Adaptive Grids for Unbounded Spherically Elliptic PDE's, SIAM J. of Scien. and Stat. Comp., Vol. 11, No. 1, 92-111, 1990, (with M. Seager).

Shock Sensitivity in PNS Solution of High Angle-of-Attack Supersonic Flow, AIAA Journal, Vol. 28, No. 3, 406-413, 1990, (with D. Cline).

Basis Function Selection and Preconditioning High Degree Finite Element and Spectral Methods, BIT 29, 794-804, 1989, (with E. Barragy).

Nodal Superconvergence and Solution Enhancement, SIAM J. of Scien. and Stat. Comp., Vol. 11, No. 2, 343-353, 1990, (with R. MacKinnon).

Least-Squares Finite Element Methods for Compressible Euler Equations, IJNMF, Vol. 10, $557-$ 568, 1990, (with B. N. Jiang).

An Improved Algorithm for Inverse Design of Thermal Problems with Multiple Materials, J. of Heat Transfer, Vol. 112, No. 2, 274-279, 1990, (with R. Shau and J. Batista).

Semiconductor Device Modeling Using Flux Upwind Finite Elements, COMPEL, Vol. 8, No. 4, 219-224, 1989, (with M. Sharma).

Periodic Viscous Flow: A Bencilmark Problem, IJNMF, Vol. 11, 87-97, 1990, (with I. Goldberg, R. McLay and L. Phinney).

Shocked Transonic Flow Calculations Using Finite Elements and a Fictitious Gas, J. Comp. Meth. in Applied Mech. and Eng., 81, 1-11, 1990, (with T. T. Pan).

Adaptive Grids for Coupled Viscous Flow and Transport, CMAME, 82, 365-383, 1990, (with K. C. Wang).

Viscous Flow and Transport with Moving Free and Reactive Surfaces, IJNME, Vol. 30, 1181 1194, 1990, (with P. Murray).

A Perturbation Analysis and Finite Element Approximate Model for Free Surface Flow Over Curved Beds, IJNME, 31, 493-507, 1991, (with R. C. Berger).

Finite Element Simulation of Phase Change Using Capacitance Methods, Journal of Numerical Heat Transfer, Part B, Vol 19, pp. 13-30, 1991, (with S. Runnels).

Bifurcation Detection Using Lanczos Method and Imbedded Subspaces, J. of Impact of Computing in Science and Engineering, 3, 76-92, 1991, (with E. Barragy).

Natural and Postprocessed Superconvergence in Semilinear Problems, Int'l J. Numer. Methods PDE's, 7, 245-259, 1991, (with S. Chow \& R. Lazarov).

Approximation of the KdV Equations by Least Squares Finite Elements, CMAME, 93, 1, 1-11, 1991, (with Y. Shen).

Preconditioners for High Degree Elements, CMAME, 93, 97-110, 1991, (with E. Barragy).

Mesh Generation/Refinement Using Fractal Concepts and Iterated Function Systems, IJNME, 33,287-305, 1992, (with S. Bova). 
Modeling Phosphorus Diffusion in Three Dimensions, IEEE Transactions on Computer Aided Design of Integrated Circuits and Systems, Vol 11, No. 4, April 1992, (with W. Richardson and B. Mulvaney).

Parallel Grid Generation/Optimization Using Block Domain Decomposition, Communications in Applied Numerical Methods, 8, 243-256, 1992, (with S. Bova).

Parallelizable Restarted Iterative Methods for Nonsymmetric Linear Systems, Part I: Theory and Part II: Parallel Implementation, International Journal of Computer Mathematics, Vol. 44, 243-290, 1992, (with W. Joubert).

\section{InPress}

Parallel-Vector Computation with High-p Element-by-Element Methods, International J. of Computer Mathematics, In Press May 1991, (with E. Barragy).

A Grid Anomaly in Quadrilateral Elements, Classroom Notes of SIAM Review, In Press October 1991, (with H. Dinh).

Superconvergence Analysis of Approximate Boundary-Flux Calculations, Numerische Mathematik, In Press June 1992, (with A. Pehlivanov, R. Lazarov and S. Chow).

Non-Linear Convective Effects on Moving Boundary AC Plasma Arcs, IEEE Transactions on Plasma Science, In Press July 1992, (with H. M. Wu).

Modeling Separated Forced Convection in Laminar Flow Past Cavities, J. of Numerical Heat Transfer, In Press July 1992, (with M. Rahman).

Stream Function Vorticity Solution Using High Degrees (p) Finite Elements and Element-byElement Techniques, CANM, In Press 1992, (with E. Barragy).

Moving Grid Finite Element Modeling of Thermal Ablation and Consolidation in Porous Media, IJNME, In Press July 1992, (with R. MacKinnon).

Iterative Solution of the Stream Function-Vorticity Equations Using a Multigrid Solver with Finite Elements, CANM, In Press September 1992, (with M. Davis).

Performance and Scalability of Finite Element Analysis for Distributed Parallel Computation, J. of Parallel and Distributed Computing, In Press October 1992, (with E. Barragy and R. Van de Geijn).

Nonlinear Dynamics of Heat Transfer Enhancement using Eddy Promoters, J. of Numerical Heat Transfer, In Press November 1992, (with M. Rahman).

Application of Conjugate Gradient Methods to Tidal Simulation, Advances in Water Resources, In Press January 1993, (with E. Barragy and R. Walters).

Shape Optimization Using Adaptive Shape Refinement, IJNME, In Press January 1993, (with H. Kohli).

\section{Submitted}

Mixing in Fluids, IJNMF, Submitted July 1990, (with Y. Shen).

Superconvergence Phenomena in Nonlinear Two Point Boundary Value Problems, Num. Meth. PDE's, Submitted April 1991, (with S. Chow).

Some Conformal Map Constructs for Numerical Grids, Num. Meth. PDE's, Submitted February 1991, (with A. Muleshkov).

Numerical Simulation of AC Plasma Arc Thermodynamics, Journal of Comp. Phy., Submitted October 1991, (with H. Wu and M. E. Oakes).

A Least Squares Finite Element Method for Viscoelastic Fluid FLow Problems, IJNMF, Submitted November 1991, (with K. C. Wang).

Computation of Hypersonic Nozzle Flows in Chemical and Thermal Nonequilibrium Using a Three-Temperature Model, AIAA Journal, Submitted November 1991, (with P. Christou).

A Scalable, Object-Oriented Finite Element Solver for Partial Differential Equations on Multicomputers, J. of Parallel and Distributed Computing, Submitted January 1992 (with J. Schmidt, V. Singh and Dennis Yelton). 
Finite Element Modelling of In Situ Vitrification, In Situ Journal, Submitted March 1992, (with R. McLay and R. MacKinnon).

Convergence Analysis of Least-Squares Mixed Finite Elements, Computing, Submitted March 1992, (with A. Pehlivanov, R. D. Lazarov and Y. Shen).

Embedded Iterative Solution of Nonlinear PDE's on the Connection Machine, Int'l Journal of High Speed Computing, Submitted April 1992, (with W. Joubert).

A Domain Decomposition Strategy for Discretized PDEs with Localized Nonlinearities, SIAM Journal, Submitted May 1992, (with S. Runnels).

Least-Squares Finite Element Approximation of Fisher's Reaction-Diffusion Equation, Num. Methods PDEs, Submitted October 1992, (with Y. Shen).

Hybrid Iterative-Extrapolation Methods: A Chebyshev-MPE Algorithm, Journal of Numerical Linear Algebra with Applications, Submitted November 1992, (with R. Abbasian).

Unsteady Shock - Separation Bubble Interaction at a Compression Corner, IJNMF, Submitted December 1992, (with M. Rahman). 


\section{Dr. David M. Young}

Dr. Young received his $\mathrm{PhD}$ from Harvard University in 1950. He has had experience as a Research Mathematician at the Computing Laboratory at the Aberdeen Proving Ground (195152), Associate Professor of Mathematics, University of Maryland (1952-55) and Manager, Mathematical Analysis Dept. of the Ramo-Wooldridge Corporation (1955-58). He joined the faculty of the University of Texas at Austin in 1958 as Professor of Mathematics. In 1967 he helped establish the Department of Computer Sciences. Dr. Young was Director of the Computation Center from 1958 until 1970. Since 1970 he has been Director of the Center for Numerical Analysis.

Dr. Young has worked extensively on the numerical solution of partial differential equations. He has been particularly concerned with effective iterative methods for solving large sparse linear systems which typically arise when discretization methods such as finite difference methods and finite element methods are used. He has been involved with the development and evaluation of various iterative algorithms using both theoretical analysis and also numerical experimentation. He has also been concerned with the actual implementation of iterative algorithms which are suitable for use on vector and parallel computers.

Dr. Young is the author of a number of research papers and reports. Selected papers and ref orts appearing since 1984 are listed below. He is the author of three books which are listed below.

For several years Dr. Young, along with Dr. Kincaid and others, has worked on the ITPACK project for developing research-oriented mathematical software for solving large sparse linear systems using iterative methods. Dr. Young was also a participant on the ELLPACK project for the development of software for solving partial differential equations.

\section{Selected Publications Since 1984}

Kinciad, D.R. and Young, D. M. [1984]. "The ITPACK Project: Past, Present, and Future," Elliptic Problem Solvers II, (Garrett Birkhoff and Arthur Schoenstadt, eds), Academic Press, New York, 53-63.

Young, D. M., Jea, K.C., and Kincaid, D. R., [1984]. "Accelerating Nonsymmetrizable Iterative Methods," in Elliptic Problems Solvers II, (G. Birkhoff and A. Schoenstadt, etd). Academic Press, New YOrk, 323-342.

Young, D. M. and Kincaid, D. R., [1984]. "On the Use of Iterative Methods with Supercomputers for Solving Partial Differential Equations," Trends in the Theory and Practice of Non-linear Analysis (V. Lakshmikantham, et.), Elsevier Science Publishers (North-Holland), The Netherlands, 455-566.

Young, D. M. and Kincaid, D. R. [1984]. "The ITPACK Software Package," in PDE Software: Modules, Interfaces and Systems, Proceedings of the International Federation of Information Processing Societies (IFIF) Working Group 2.5 on Numerical Software (B. Engquist, ed), Elsevier Science Publishers (North-Holland), Amsterdam, The Netherlands, 193-206.

Kincaid, D.R., Carey, G. F., Oppe, T. C., Sepehrnoori, K., and Young, D. M. [1984]. "Combining Finite Element and Iterative Methods for Solving Partial Differential Equations on Advanced Compuer Architectures," Advances in Computer Methods for Partial Differential Equations IV (R. Vichnevetsky and R. S. Stepleman, eds.) publ. IMACS Dept. of Computer Science, Rutgers University, New Brunswick, N.J.

Kincaid, D.R., Oppe, T. C. Respess, J.R., and Young, D.M. [1985]. "Chapter 7: ITPACK Solution Modules", in Solving Elliptic Problems Using ELLPACK (J.R. Rice and R. F. Boisvert, eds.), Springer-Verlag, New York, 237-258.

Young, D. M., Oppe, T. C., Kincaid, D. R. and Hayes, L. J. [1985]. "On the Use of Vector Computers for Solving Sparse Linear Systems," Report CNA-199, Center for Numerical Analysis, University of Texas, Austin, TX. 
Young, D. M., Kincaid, D. R. and Oppe, T. C. [1986]. "Vectorized Iterative Methods for Partial Differential Equations," Communications in Applied Numerical Methods, Vol 2, 289-296.

Joubert, Wayne, and Young, David M. [1986]. "Necessary and Sufficient Conditions for the Simplification of Generalized Conjugate Gradient Algorithms," Linear Algebra and its Applications, 86/87, 449-485.

Kincaid, Daivd R., Oppe, Thomas C., and Young, David M. [1986]. "Vector Computations for Sparse LInear Systems," SIAM Journal of Alg and Disc. Meth., 7, 99-112.

Young, David M. and Mai, Tsun-zee [1987]. "Iterative Algorithms and Software for the Solution of Large Sparse Linear Systems." Communications in Applied Numerical Methods, 4, 435456.

Kincaid, David R. and Young, David M. [1988]. "A Brief Review of the ITPACK Project," Report CNA-217, Center for Numerical Analysis, The University of Texas, Austin, TX, Journal of Computational and Applied Mathematics, 24, 33-54.

Jea, Kang C. and Young, David M. [1988]. "On the Effectiveness of Adaptive Chebyshev Acceleration for Solving Systems of Linear Equations," Journal of Computational and Applied Mathematics, 34, 33-54.

Adams, Loyce M., LeVeque, Randall J., and Young, D. M. [1988]. "Analysis of the SOR Iteration for the 9-point Laplacian," SIAM J. Numer. Anal.., 25, 1156-1180.

Carey, G. F., Kincaid, Dr.R., Sepehrnoori, K. and Young, D. M. [1988]. "Vector and Parallel Iterative Solution of Large Sparse Systems for PDEs." Proceeding of the Cray Supercomputer Conference, Minneapolis, Minnesota, October 1988.

Mai, Tsun-zee and Young, D. M. [1988]. "A Dual Adaptive Procedure for the Automatic Determination of Iteration Parameters for Chebyshev Acceleration," Report CNA-224, Center for Numerical Analysis, The University of Texas, Austin, Texas.

Young, David M., Jea, Kang C. and Mai, Tsun-Zee [1988]. "Preconditioned Conjugate Gradient Algorithms and Software for Solving Large Sparse LInear Systems," Appeared in LInear Algebra in Signals, Systems and Control (B. N. Datta, ed.) SIAM, Philadelphia, PA.

Young, David M. [1989]. "A Historical Overview of Iterative Methods," Computer-Physics Communications, 53, 1-17.

Jea, Kang C., and Young, David M. [1991] "Commentaries on Three Papers of Cornelius Lanczos," Report CNA-252, Center for Numerical Analysis, University of Texas at Austin.

Kincaid, David R. and Young, David M. "Stationary Second-degree Iterative Methods and Recurrences." In R. Beauwens and R. De Groen, editors, Iterative Methods in Linear Algebra, pages 27-47. Elsevier Science Publishers B.V. (North-Holland), New York, 1992.

Young, David M. [1989], "The Search for "High Level" Parallelism for Iterative Sparse Linear System Solvers", Chapter 7 of Parallel Supercomputing: Methods, Algorithms and Applications, edited by Graham F. Carey, John Wiley and Sons, Ltd.

Young, David M. [1990], "A Historical Review of Iterative Methods", appeared in A History of Scientific Computing, edited by Stephen G. Nash, History Series, ACM Press, New York, 180-194.

Young, David M. and Mai, Tsun-zee [1990], "The Search for Omega", Chapter 17 of Iterative Methods for Large Linear Systems, edited by David R. Kincaid and Linda J. Hayes, Academic Press, Inc.

Young, David M., and Vona, Bi Roubolo [1990]. "Parallel Multilevel Methods," Report CNA243, Center for Numerical Analysis, University of Texas at Austin. (To appear in the Proceedings of a conference held at Purdue University in November 1989 in honor of Professor S. D. Conte.)

Young, David M., and Vona, Bi Roubolo [1992]. "On the Use of Rational Iterative Methods for Solving Large Sparse Linear Systems," Applied Numerical Mathematics, 10, 261-278.*

Young, David M. and Kincaid, David R. "Linear Stationary Second-Degree Methods for the Solution of Large Linear Systems," In Th. M. Rassias, H. M. Srivasiava, and A. Yanushauska, editors, Topics in Polynomials of One and Several Variables and their Applications. World Scientific Publ. Co., Singapore, to appear 1992. 


\section{Dr. David R. Kincaid \\ Co-Investigator}

Dr. Kincaid received the B.S. (cum laude) degree from Lamar University and the M.A. and Ph.D. degrees from The University of Texas at Austin. He taught in the Computer Science Department at Purdue University before returning to The University of Texas at Austin. Currently, Dr. Kincaid is a Research Scientist in the Computation Center, a Senior Lecture in the Computer Sciences Department, and the Associate Director of the Center for Numerical Analysis. $\mathrm{He}$ is also a member of the Texas Institue of Computational Mechanics.

Dr. Kincaid is actively involved in numerical analysis research, especially numerical linear algebra and iterative methods, with emphasis on the use of high performance computers. His participation in the project includes supervising the development of software packages based on iterative algorithms, learning and utilizing parallelization techniques and testing the resulting software on parallel architectures.

Dr. Kincaid has had extensive experience in programming and in the supervision of programming activities. For example, he was involved in the development of three well-known software packages: BLAذ, Basic Linear Algebra Subprograms; ITPACK, an iterative package for solving symmetric positive definite linear systems; and MSPCG, an nonsymmetric preconditioned conjugate gradient package. Recently, he has gained considerable experience on supercomputers with the adaptation of software for use on vector and parallel computers. Dr. Kincaid was a contributor to the ELLPACK package (Purdue University) for the development of software for solving partial differential equations.

Drs. David Kincaid and Ward Cheney have written two textbooks: an advanced textbook Numerical Analysis: Mathematics of Scientific Computing and an introductory textbook Numerical Mathematics and Computing, 2nd Edition.

Dr. Kincaid is the author of a number of research papers and technical report. Those published since 1984 are listed below.

\section{Publication Since 1984}

Ward Cheney and David Kincaid. Numerical Mathematics and Computing. Brooks/Cole Publishing Co., Pacific Grove, CA, 2nd edition, 1985. 562 pages.

David Kincaid and Ward Cheney. Numerical Analysis: Mathematics of Scientific Computing. Brooks/Cole Publishing Co., Pacific Grove, CA, 1990. 690 pages.

David R. Kincaid, Graham F. Carey, Kamy Sepehrnoori, and David M. Young. Vector and parallel iterative solution of large sparse systems for PDE's. In Science and Engineering on Cray Supercomputers, pages 25-44. Cray Research, Inc., Minneapolis, MN, 1988.

David R. Kincaid, Graham F. Carey, Kamy Sepehrnoori, and David M. Young. Vector and parallel iterative solution of large sparse systems for PDEs. Re- 
port CNA-222, University of Texas at Austin, Center for Numerical Analysis, August 1988.

David R. Kincaid and Matthew D. Fassiotto. Itpack software and parallelization. Report CNA-255, University of Texas at Austin, Center for Numerical Analysis, June 1992.

David R. Kincaid and Linda J. Hayes, editors. Iterative Methods for Large Linear Systems. Academic Press, New York, 1989. 318 pages.

David R. Kincaid and Thomas C. Oppe. A parallel algorithm for the general LU factorization. Communications in Applied Numerical Methods, 4(3):349$359,1988$.

David R. Kincaid and Thomas C. Oppe. Some parallel algorithms on the four processor Cray X-MP4 supercomputer. Report CNA-220, University of Texas at Austin, Center for Numerical Analysis, May 1988.

David R. Kincaid and Thomas C. Oppe. Some parallel algorithms on the four processor cray x-mp4 supercomputer. In Graham F. Carey, editor, Parallel Supercomputing: Methods, Algorithms and Applications, chapter 5, pages 121-134. Wiley, New York, 1989.

David R. Kincaid and Thomas C. Oppe. Recent vectorization and parallelization of ITPACK. In O. Axelsson and L. Y. Kolotilina, editors, Preconditioned Conjugate Gradient Methods, pages 58-78. Springer-Verlag, New York, 1990. Lecture Notes in Mathematics 1457.

David R. Kincaid, Thomas C. Oppe, and Wayne D. Joubert. An overview of NSPCG: A nonsymmetric preconditioned conjugate gradient package. Report CNA-228, University of Texas at Austin, Center for Numerical Analysis, October 1988.

David R. Kincaid, Thomas C. Oppe, and Wayne D. Joubert. An introduction to the NSPCG software package. International Journal of Numerical Methods in Engineering, 27(3):589-608, 1989.

David R. Kincaid, Thomas C. Oppe, and Wayne D. Joubert. An overview of NSPCG: A nonsymmetric preconditioned conjugate gradient package. In Daniel L. Boley, Donald G. Truhlar, Youcef Saad, Robert E. Wyatt, and Lee A. Collins, editors, Practical Iterative Methods for Large Scale Computations, pages 283-293. North-Holland, Amsterdam, 1989. (Reprinted from Computer Physics Communications, 53(3):283-293, 1989.).

David R. Kincaid, Thomas C. Oppe, and David M. Youngi. Vector computations for sparse linear systems. SIAM J. Alg. Disc. Malh., 7(1):99-112, 1986.

David R. Kincaid, Thomas C. Oppe, and David M. Young. Vectorized iterative methods for partial differential equaticas. Communications in Applied Numerical Methods, 2(3):289-296, 1986. 
David R. Kincaid, Thomas C. Oppe, and David M. Young. ITPACKV 2D user's guide. Report CNA-232, University of Texas at Austin, Center for Numerical Analysis, May 1989.

David R. Kincaid, Thomas C. Oppe, and David M. Young. Are there iterative BLAS? Report CNA-240, University of Texas at Austin, Center for Numerical Analysis, February 1990.

David R. Kincaid and Malathi Ramdas. Paralleling ITPACKV 2D. In J. J. H. Miller and R. Vichnevetsky, editors, IMACS '91: Proceedings of the 19th IMACS World Congress on Computational and Applied Mathemalics, volume 2, pages 686-687. Criterion Press, Dublin, Ireland, 1991.

David R. Kincrid and David M. Young. The ITPACK project: Past, present, and future. In Garrett Birkhoff and Arthur Schoenstadt, editors, Elliptic Problem Solvers II, pages 53-63. Academic Press, New York, 1984.

David R. Kincaid and David M. Young. A tutorial on finite difference methods and ordering of mesh points. In H. S. Stone and S. Winkler, editors, Fall Joint Computer Conference Proceedings, pages 556-559. IEEE Computer Society Press, Washington, D.C., 1986.

David R. Kincaid and David M. Young. A brief review of the ITPACK project. Journal of Computational and Applied Mathematics, 24:121-127, 1988.

David R. Kincaid and David M. Young. Linear stationary second-degree methods for solution of large linear systems. Report CNA-244, University of Texas at Austin, Center for Numerical Analysis, July 1990.

David R. Kincaid and David M. Young. Stationary second-degree iterative methods and recurrences. Report CNA-250, University of Texas at Austin, Center for Numerical Analysis, February 1991.

David R. Kincaid and David M. Young. Stationary second-degree iterative methods and recurrences. In R. Beauwens and R. De Groen, editors, Iterative Methods in Linear Algebra, pages 27-47. Elsevier Science Publishers B.V. (North-Holland), New York, 1992.

Thomas C. Oppe, Wayne D. Joubert, and David R. Kincaid. NSPCG user's guide, version 1.0: A package for solving large sparse linear systems by various iterative methods. Report CNA-216, University of Texas at Austin, Center for Numerical Analysis, April 1988.

Thomas C. Oppe and David R. Kincaid. Numerical experiments with a parallel conjugate gradient method. In R. Vichnevetsky and R. S. Stepleman, editors, Advances in Computer Methods for Partial Differential Equations, volume VI, pages 369-374. IMACS, Department of Compliter Sciences, Rutgers University, New Brunswick, NJ, 1987.

Thomas C. Oppe and David R. Kincaid. Parallel LU-isctorization algorithms for dense matrices. In E. N. Houstis, T. S. Papistheodorou, and C. D. 
Polychronopoulos, editors, Supercomputing, pages 576-594. Springer-Verlag, New York, 1988. Lecture Notes in Computer Science 297.

Malathi Ramdas and David R. Kincaid. Parallelizing ITPACKV 2D for the Cray Y-MP. Report CNA-249, University of Texas at Austin, Center for Numerical Analysis, February 1991.

Malathi Ramdas and David R. Kincaid. Parallelizing ITPACK 2D for the Cray Y-MP. In R. Beauwens and R. De Groen, editors, Iterative Methods in Linear Algebra, pages 323-337. Elsevier Science Publishers B.V. (North-Holland), New York, 1992.

Marilyn Santiago and David R. Kincaid. Using cyclic reduction on a parallel computer to improve the performance of an underwater sound implicit finite difference model. Computers in Mathematical Applications, 21(5):83-94, 1991.

David M. Young, David R. Kincaid, and Kang C. Jea. Accelerating nonsymmetric iterative methods. In Garrett Birkhoff and Arthur Schoenstadt, editors, Elliptic Problem Solvers II, pages 323-342. Academic Press, New York, NY, 1984. 


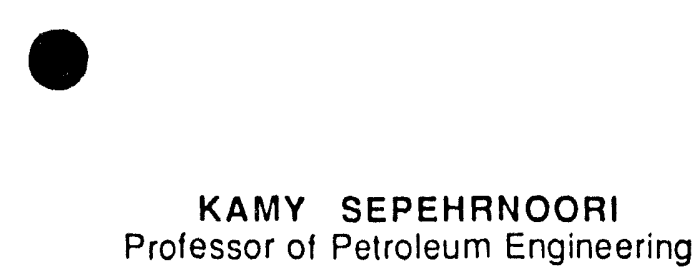

\title{
EDUCATION
}

B.S. (Mechanical Engineering) The University of Texas, 1973; M.S. (Engineering) The University of Texas, 1974; Ph.D. (Petroleum Engineering) The University of Texas, 1977.

\section{EDUCATIONAL EXPERIENCE}

1989-present Frank W. Jessen Professor in Petroleum Engineering; 1985-1989 Associate Professor of Petroleum Engineering, The University of Texas at Austin; $1981-1985$ Assistant Professor of Petroleum Engineering. The University of Texas at Austin; 1982-Summer Visiting Professor, Department of Petroleum Engineering. The University of Southwestern Louisiana; 1975-Fall Teaching Assistant, Department of Aerospace Engineering and Engineering Mechanics, The University of Texas at Austin.

\section{OTHER EXPERIENCE}

1983 - Summer Summer job training at Dresser Allas and Shell Oil Company - Houston; 1978 1981 Research Engineer - Scientist Associate, Center for Energy Studies and Petroleum Engineering Department. The University of Texas at Austin; 1979 - Summer Research Associate, Department of Petroleum and Geological Engineering University of Oklahoma; 1973 1977 Research Engineer - Scientist Assistant, Bureau of Engineering Research, The University of Texas at Austin.

\section{HONORS AND AWARDS}

Honor Roll, College of Engineering, The University of Texas. Sigma Gamma Tau, Phi Theta Kappa, Phi Kappa Phi, Recipient of Digital Equipment Corporation Grant for the Development of Computer Graphics in the Petroleum Engineering Department, 1982. Award of Excellence, Halliburton Education Foundation, September, 1984. Recipient of Sun Exploration and Production Company Centennial Fellowship, 1983-1989. GIGAFLOP Performance Award from Cray Research, Inc., 1989. Frank W. Jessen Professor in Petroleum Engineering, 1989-present.

\section{MEMBERSHIPS}

Society for Industrial and Applied Mathematics (SIAM) - Society of Petroleum Engineers (SPE)

\section{UNIVERSITY ASSIGNMENTS}

Computer Committee, Dept. of Pet. Eng. - Admission Committee, Dept. of Pet. Eng., Graduate Studies Committee, Dept. of Pet. Eng., Chairman, Library Committee, Dept. of Pet. Eng., Engineering Computer Committee.

\section{NON-UNIVERSITY ASSIGNMENTS \\ General Editor, In Situ}

\section{CONSULTING}

Digital Equipment Corporation

\section{PUBLICATIONS}

Dr. Sepehrnoori has published over 100 technical articles and reports in his research related areas.

\author{
AREAS OF INTEREST \\ Reservoir Simulation \\ Computational Methods \\ Applied Mathematics \\ Enhanced Oil Recovery \\ Database Application in Petroleum Engineering \\ Vector/Parallel Computations
}




\section{MOST RECENT PUBLICATIONS}

"An Expert System for Miscible Gas Flooding," with S.A. Khan and G.A. Pope, 10 appear in SPE Computer Applications, February 1993.

"An Expert System to Select Acid Gas Treating Processes for Natural Gas Processing Plants," with H. Kurimura and G.T. Rochelle, accepted tor publication by J. Gas Separation and Purification, 1992.

"Application of Higher-Order Flux-Limited Methods in Compositional Simulation," with ن. Liu, M. Delshad, and G.A. Pope, submit!ed to Transport in Porous Media, November 1992.

"The Effect of Four Geostatistical Methods on Reservoir Description and Flow Mechanism," with S.G. Ghori, A. Ouenes, G.A. Pope, and J.P. Heller, SPE 24755 to be presented at the SPE 67th Annual Technical Conterence \& Exhibition, October 4-7, 1992, Washington, DC.

"Simulation of a Successful Polymer Flood in the Chateaurenard Field," with S. Takagi, A. Putz, and G.A. Pope, SPE 24931 to be presented at the SPE 67th Annual Technical Conference \& Exhibition, October 4-7, 1992, Washington, DC.

"Simulation of Carbon Dioxide Flooding Using Horizontal Wells," with M.T. Lim, S.A. Khan, and G.A. Pope, SPE 24929 to be presented at the SPE 67th Annual Technical Conference \& Exhibition, October 4-7, 1992, Washington, DC.

"Corrosion in Alkanolamine Systems for Acid Gas Treating," with H. Kurimura and G.T. Rochelle, submitted to J. Materials Performance, July 1992.

"Simulation of the Performance of Surfactant Enhanced Remediation of DNAPL Contaminated Aquifers," with D.C. McKinney, C.L. Brown, M. Jin, G.A. Pope, and L.M. Abriola, Proceedings of the 3rd International Conference on Ground Water Quality Research, June 1992.

"Simulation of Sandstone Acidizing of a Damaged Perforation," with C-M. Lea and A.D. Hill, SPE Production Engineering, May 1992.

"A Single-Well Tracer Test to Estimate Weltability," with L.E.A. Ferreira, F. Descant, M. Delshad, and G.A. Pope, SPE 24136 presented at the SPE/DOE 8th Symposium on Enhanced Oil Recovery, Tulsa, OK, April 22-24, 1992.

"Fluid Characterization of Three-Phase $\mathrm{CO}_{2} /$ Oil Mixtures," with S.A. Khan and G.A. Pope, SPE24130 presented at the SPE/DOE 8th Symposium on Enhanced Oil Recovery, Tulsa, OK, April 22-24, 1992.

"Analysis of Tertiary Injectivity of Carbon Dioxide," with M.K. Roper, Jr. and G.A. Pope, SPE 23974 presented at the SPE/DOE 8th Symposium on Enhanced Oil Recovery, Tulsa, OK, April 22-24, 1992, and the SPE Permian Basin Oil and Gas Recovery Conference, Midland, TX, March 18-20, 1992.

"Interpretation of a $\mathrm{CO}_{2}$ WAG Injectivity Test in the San Andres Formation Using a Compositional Simulator," with M.K. Roper, Jr., C.T. Cheng, J. Varnon, and G.A. Pope, SPE 24163 presented at the SPE/DOE Eighth Symposium on Enhanced Oil Recovery, Tulsa, OK, April 22-24, 1992.

"Reformation of Xanthan/Chromium Gels After Shear Degradation," with J.S. Tsau, J. Liang, and A.D. Hill, SPE Reservoir Engineering. February 1992.

"Oil Recovery From Naturally Fractured Reservoirs by Steam Injection Methods," with M.A. Miller, J.C. Reis, O. Trevisan, and J. Chen, Proceedings of the IEA Collaborative Project on Enhanced Oil Recovery Workshop and Symposium, Bath, UK, Oclober 28-30, 1991. 
"Carbon Dioxide Flow Patterns Under Multiphase Flow, Heterogeneous, Field Scale Conditions," with Y. Chang, M.T. Lim, and G.A. Pope, SPE 22654 presented at the SPE 66th Annual Technical Conference and Exhibition, Dallas, TX, October 6-9, 1991.

"The Elfect of Fluid Diversion on the Acid Stimulation of a Perforation," with C.-M. Lea and A.D. Hill, SPE 22853 presented at the SPE 66th Annual Technical Conference and Exhibition, Dallas, TX, Oclober 6-9, 1991.

"High Resolution Monotonic Schemes for Reservoir Fluid Flow Simulation," with A. Datta Gupta, L.W. Lake, G.A. Pope, and M.J. King, In Situ, 15(3), 1991.

"A Study of the Permeability-Reducing Characteristics of Polyacrylamide/ Chromium Gels," with J. Liang, J.S. Tsau, and A.D. Hill, In Situ, 15(3), 1991.

"Three-Phase Gas/Oil/Brine Relative Permeabilities Measured Under Carbon Dioxide Flooding Conditions," with D. Dria and G.A. Pope, SPE 20184 accepted for publication in SPE Reservoir Engineering, April, 1991.

"Design of the $\mathrm{HCl}$ Preflush in Sandstone Acidizing," with A.D. Hill and P.Y. WU, SPE 21720 presented at the SPE Production Operations Symposium, Oklahoma City, OK, April 7-9, 1991.

"Comparisons of Counter-Current Imbibition Transfer Functions in Dual Porosity Models of Naturally Fractured Reservoirs," with J. Chen and M.A. Miller, In Situ, 15(2), 1991.

"Simulation of Heterogeneous Sandstone Experiments Characterized Using C.T. Scanning," with S. Ganapathy, D. Wreath, M.T. Lim, B. Rouse, and G.A. Pope, SPE 21757 presented at the SPE Western Regional Meeting, Long Beach, CA, March 20-22, 1991.

"Development of a Thermodynamically Consistent, Fully Implicit, Equation-of-State, Compositional Steamflood Sirnulator," with K. Brantferger and G.A. Pope, SPE 21253 presented at the SPE Symposium on Reservoir Simulation, Anaheim, CA, February 17-20, 1991.

"Analysis of Field Tracers for Reservoir Description," with S.B. Allison and G.A. Pope, J. of Petroleum Science and Engineering, 5(2), January, 1991.

"Benchmark of UTCHEM Reservoir Simulator on Various Cray Computers," with G. Shiles and G.A. Pope, Communications in Applied Numerical Methods, 7 (1), January, 1991.

"The Elfects of Gravity on Micellar-Polymer Flooding Under North Sea Conditions: A Simulation Study," with M. Shook and G.A. Pope, In Situ, 15(1), January, 1991.

"Application of Higher-Order Methods in Compositional Simulation," with N. Saad and G.A. Pope, SPE Reservoir Engineering, November, 1990.

"A Higher-Order Finite Difference Compositional Simulator," with Y. Chang and G.A. Pope, J. of Petroleum Science and Engineering, 5(1), November 1990.

"Numerical Simulation of Interwell Tracers," with G.A. Pope and G. Shiles, accepted by in Situ, October 1990.

"Petroleum Reservoir Simulation on the CRAY Y-MP," with G. Shiles and G.A. Pope, Proceedings of the Fifth International Conference of Science and Engineering on Supercomputers, E.J. Pitcher (ed.), Springer-Verlag, 1990.

"Dependence of Polymer Apparent Viscosity on the Permeable Media and Flow Conditions," with D. Wreath and G.A. Pope, In Situ, 14(3), 1990. 


\section{APPENDIX I}

\section{UTCHEM SIMULATOR AND GOVERNING EQUATIONS}

In UTCHEM, the material balance equations are solved for up to nineteen components: water, oil, surfactant, polymer, anions, divalent cations, cosurfactant-1, cosurfactant-2, three tracers, sodium dichromate, thiourea, trivalent chromium, gel, hydrogen, carbon, silicon, and organic acid species. These components may form up to three phases-aqueous, oleic, and microemulsion-depending on the relative amounts and effective salinity of the phase environment.

The major physical phenomena modeled in the simulator are phase density, phase viscosity, phase behavior, dispersion, adsorption, interfacial tension, relative permeability, capillary pressure, capillary trapping, cation exchange, and polymer properties. The latter includes permeability reduction, inaccessible pore volume, and shear-thinning. Depending on the process and components injected, various chemical reactions to form gel or precipitation-dissolution reactions are modeled.

Next, we present the material balance equations and the pressure equation used in the simulator as well as a brief description of the numerical schemes.

\section{Material balance equations}

The material balance equations solved in UTCHEM are given below. A detailed description of these equations can be found in Datta (jupta et al. (1986), Pope et al. (1990), and Bhuyan et al. (1990).

$$
\begin{aligned}
& \frac{\partial}{\partial t}\left[\phi \tilde{c}_{i}\left(1+c_{i}^{\circ} \Delta P\right)\right]+ \\
& +\frac{\partial}{\partial x}\left\{\sum_{j=1}^{n_{p}}\left(1+c_{i}^{o} \Delta P\right)\left[c_{i j} u_{x j}-\phi s_{j}\left(K_{x x i j} \frac{\partial c_{j i}}{\partial x}+K_{x y i j} \frac{\partial c_{i j}}{\partial y}+K_{x z i j} \frac{\partial c_{i i}}{\partial z}\right)\right]\right\}+ \\
& +\frac{\partial}{\partial y}\left\{\sum_{j=1}^{n_{p}}\left(1+c_{i}^{\circ} \Delta P\right)\left[c_{i j} u_{y j}-\phi s_{j}\left(K_{y x i j} \frac{\partial c_{i i}}{\partial x}+K_{y y i j} \frac{\partial c_{i j}}{\partial y}+K_{y z i j} \frac{\partial c_{i j}}{\partial z}\right)\right]\right\}+ \\
& +\frac{\partial}{\partial z}\left\{\sum_{j=1}^{n_{p}}\left(1+c_{i}^{\circ} \Delta P\right)\left[c_{i j} u_{z j}-\phi S_{j}\left(K_{z x i j} \frac{\partial c_{i i}}{\partial x}+K_{z y i j} \frac{\partial c_{i j}}{\partial y}+K_{z z i j} \frac{\partial c_{i j}}{\partial z}\right)\right]\right\}= \\
& \mathrm{Q}_{\mathrm{i}} \quad \mathrm{i}=1, \ldots, \mathrm{n}_{\mathrm{c}},
\end{aligned}
$$


0
$\bar{c}_{i}=\left(1-\sum_{i=1}^{n_{c}} \bar{c}_{i}\right) \sum_{j=1}^{n_{p}}\left(c_{i j} s_{j}\right)+\bar{c}_{i}$.

where $\Delta P=P_{j}-P_{R}$ and $\bar{c}_{i}=\left(1-\sum_{i=1}^{n_{c}} \bar{c}_{i}\right) \sum_{j=1}^{n_{p}}\left(c_{i j} S_{j}\right)+\bar{c}_{i}$.

The overall material balance equation is obtained by summing the material balance equation (Eq. 1) over all components:

$$
\begin{aligned}
& \phi c_{t} \frac{\partial p}{\partial t}+\frac{\partial}{\partial x} \sum_{j=1}^{n_{p}}\left[u_{x j} \sum_{i=1}^{n_{c}}\left(1+c_{i}^{o} \Delta P\right) c_{i j}\right]+ \\
& +\frac{\partial}{\partial y} \sum_{j=1}^{n_{p}}\left[u_{y j} \sum_{i=1}^{n_{c}}\left(1+c_{i}^{\circ} \Delta P\right) c_{i j}\right]+ \\
& +\frac{\partial}{\partial z} \sum_{j=1}^{n_{p}}\left[u_{z j} \sum_{i=1}^{n_{c}}\left(1+c_{i}^{\circ} \Delta P\right) c_{i j}\right]=\sum_{i=1}^{n_{c}} Q_{i}, \\
& \text { where } c_{t}=c_{f}+\sum_{i=1}^{n_{c}} c_{i}^{\circ} \tilde{c}_{i} .
\end{aligned}
$$

\section{Pressure equation}

The pressure equation is obtained by substituting the multiphase flow version of Darcy's law given below for the flux terms $u_{x j}, u_{y j}$, and $u_{z j}$, in (2):

$$
\vec{u}_{j}=-\vec{k} \lambda_{r j}\left(\vec{\nabla} P_{j}-\gamma_{j} \vec{\nabla} D\right)
$$

where $\vec{k}$ is taken to be a diagonal tensor in UTCHEM. By using the definition of capillary pressure,

$\mathrm{P}_{\mathrm{c} j \not 1} \mathrm{j}=\mathrm{P}_{\mathrm{j} \not}-\mathrm{P}_{1}$ for $\mathrm{j} \notin=2,3$ we can write the resulting equation in terms of a reference phase (aqueous) pressure: 


$$
\begin{aligned}
& \phi c_{\mathrm{t}} \frac{\partial \mathrm{P}_{1}}{\partial \mathrm{t}}+\vec{\nabla} \cdot \overrightarrow{\overrightarrow{\mathrm{k}}} \cdot \lambda_{\mathrm{rTc}} \vec{\nabla} \mathrm{P}_{1}=-\vec{\nabla} \cdot \sum_{\mathrm{j}=1}^{\mathrm{n}_{\mathrm{p}}} \overrightarrow{\overrightarrow{\mathrm{k}}} \cdot \lambda_{\mathrm{rjc}} \vec{\nabla} \mathrm{D}+ \\
& +\vec{\nabla} \cdot \sum_{j=2}^{n_{p}} \overrightarrow{\vec{k}} \cdot \lambda_{r j c} \vec{\nabla} P_{c j 1}+\sum_{i=1}^{n_{c}} Q_{i} \\
& \text { where } \lambda_{\text {rjc }}=\lambda_{\text {rj }} \sum_{i=1}^{n_{c}}\left(1+c_{1}^{\rho} \Delta P\right) c_{i j} \\
& \text { and } \lambda_{r T c}=\sum_{j=1}^{n_{p}} \lambda_{r j} \sum_{i=1}^{n_{c}}\left(1+c_{i}^{\circ} \Delta P\right) c_{i j}
\end{aligned}
$$




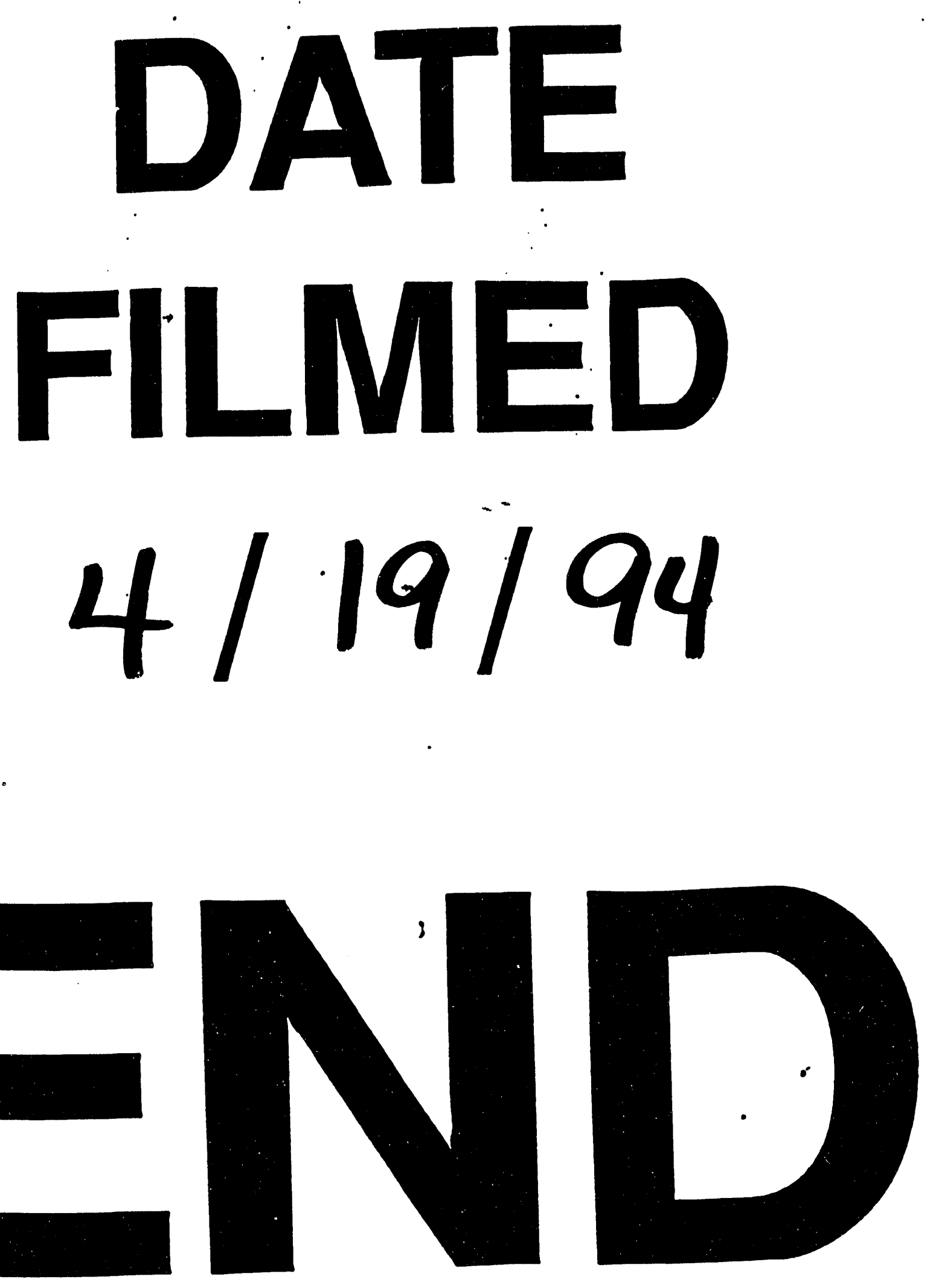



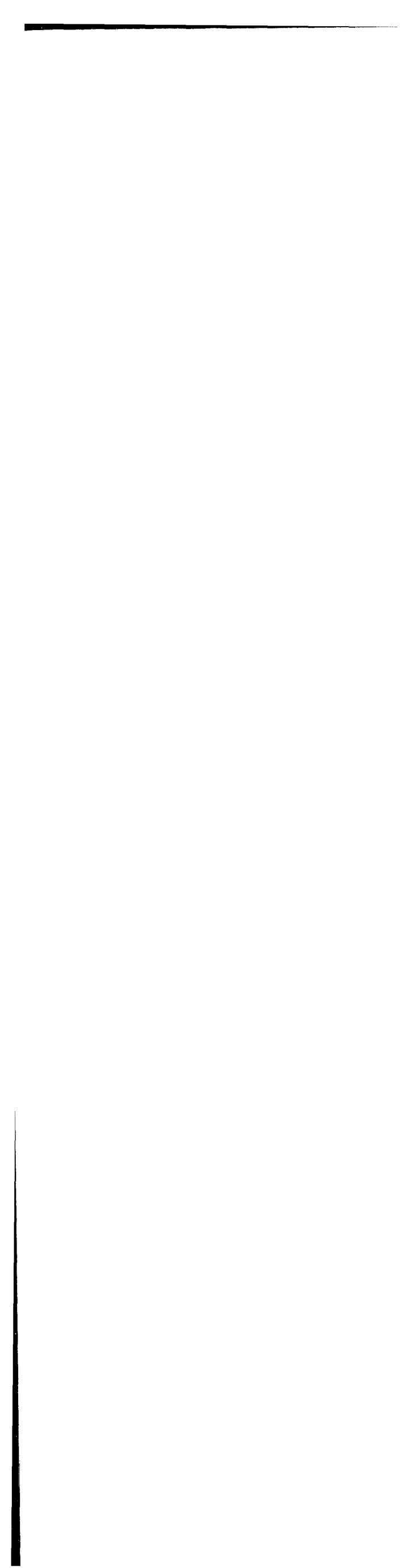

, 